\title{
The Lhx2 Transcription Factor Controls Thalamocortical Axonal Guidance by Specific Regulation of Robo1 and Robo2 Receptors
}

\author{
Paula Marcos-Mondéjar, ${ }^{1}$ Sandra Peregrín, ${ }^{1}$ James Y. Li, ${ }^{2}$ Leif Carlsson, ${ }^{3}$ Shubha Tole, ${ }^{4}$ \\ and Guillermina López-Bendito ${ }^{1}$ \\ ${ }^{1}$ Instituto de Neurociencias de Alicante, Consejo Superior de Investigaciones Científicas and Universidad Miguel Hernández, 03550 Sant Joan d'Alacant, \\ Spain, ${ }^{2}$ Department of Genetics and Developmental Biology, University of Connecticut Health Center, 263 Farmington Avenue, Farmington, Connecticut \\ 06030-6403, 3'Umeå Center for Molecular Medicine, Umeå University, 90187 Umeå, Sweden, and ${ }^{4}$ Department of Biological Sciences, Tata Institute of \\ Fundamental Research, Colaba, Mumbai 400 005, India
}

The assembly of neural circuits is dependent upon the generation of specific neuronal subtypes, each subtype displaying unique properties that direct the formation of selective connections with appropriate target cells. Actions of transcription factors in neural progenitors and postmitotic cells are key regulators in this process. LIM-homeodomain transcription factors control crucial aspects of neuronal differentiation, including subtype identity and axon guidance. Nonetheless, their regulation during development is poorly understood and the identity of the downstream molecular effectors of their activity remains largely unknown. Here, we demonstrate that the $L h \times 2$ transcription factor is dynamically regulated in distinct pools of thalamic neurons during the development of thalamocortical connectivity in mice. Indeed, overexpression of $L h x 2$ provokes defective thalamocortical axon guidance in vivo, while specific conditional deletion of $L h \times 2$ in the thalamus produces topographic defects that alter projections from the medial geniculate nucleus and from the caudal ventrobasal nucleus in particular. Moreover, we demonstrate that $L h \times 2$ influences axon guidance and the topographical sorting of axons by regulating the expression of Robo1 and Robo2 guidance receptors, which are essential for these axons to establish correct connections in the cerebral cortex. Finally, augmenting Robol function restores normal axon guidance in Lhx2-overexpressing neurons. By regulating axon guidance receptors, such as Robo1 and Robo2, Lhx2 differentially regulates the axon guidance program of distinct populations of thalamic neurons, thus enabling the establishment of specific neural connections.

\section{Introduction}

Proper development of neural connections in the brain requires the guidance of axons to their final destination through the coordinated activity of growth factors and guidance cues expressed along the pathway they follow. Because axon guidance typically involves a complex set of instructions, the generation of specific connectivity requires axons to respond to spatiotemporal guidance cues in a highly regulated manner. Genetic studies in mice have demonstrated the role of LIM homeodomain (LIM-HD)

\footnotetext{
Received Nov. 23, 2011; revised Jan. 20, 2012; accepted Jan. 30, 2012

Author contributions: G.L.-B. designed research; P.M.-M. and G.L.-B. performed research; S.P., J.Y.L., L.C., and S.T. contributed unpublished reagents/analytic tools; P.M.-M. and G.L.-B. analyzed data; G.L.-B. wrote the paper.

This work was supported by grants from the Spanish Ministry of Science and Innovation (BFU2006-00408/BFI, BFU2009-08261, and Consolider CSD2007-00023). P.M.-M. was supported by a Formación de Personal Investigador Fellowship from the Spanish Ministry of Science and Innovation. We thank L. Bluy, N. García, and E. San Martín for their excellent technical assistance, M. Dominguez for the use of the quantitative PCR equipment, S. Nóbrega and J. Galcerán for advice on the quantitative PCR and ChIP experiments, and Marc Tessier-Lavigne (Genentech) for providing the original Robo1 construct. We are also grateful to members of the López-Bendito and Herrera laboratories for stimulating discussions and comments. We thank Katja Brose for providing Robo 1 and Robo2 CDNA probes, Sonia Garel for providing the Robo 3 cDNA probe, Sylvie Retaux for providing the Lhx2 CDNA probe, and John Rubenstein for providing the Ngn2 and Gbx2 CDNA probes.

Correspondence should be addressed to Dr. Guillermina López-Bendito, Instituto de Neurociencias de Alicante, CSIC \& Universidad Miguel Hernández, 03550 Sant Joan d'Alacant, Spain. E-mail: g.lbendito@umh.es.

DOI:10.1523/JNEUROSCI.5851-11.2012

Copyright $\odot 2012$ the authors $\quad 0270-6474 / 12 / 324372-14 \$ 15.00 / 0$
}

proteins in determining the identity of columnar motor neurons (Jurata et al., 2000; Kania et al., 2000; Lee and Pfaff, 2001; Kania and Jessell, 2003). Moreover, a specific combination of transcription factors from the LIM-HD family regulates the topographic targeting of distinct pools of axons to given muscles in the limb mesenchyma (Sharma et al., 1998; Kania et al., 2000). In recent years, several candidate genes, such as the tyrosine kinase EphA4, have been identified as potential downstream effectors of these transcription factors.

The development of thalamocortical connections is characterized by the segregation of thalamic neurons into the distinct mediolateral and caudorostral nuclei that projects topographically to different cortical areas (López-Bendito and Molnár, 2003; Molnár et al., 2003). While the activity of transcription factors expressed at the origin, along the pathway, or in the target regions influences the development of this projection (Tuttle et al., 1999; Garel et al., 2002; Jones et al., 2002; López-Bendito et al., 2002; Pratt et al., 2002; Seibt et al., 2003), it remains unclear how distinct pools of thalamocortical-projecting neurons are topographically specified and which transcription factors regulate the growth of their axons. The $L h \times 2$ transcription factor is a member of the LIM-HD family of proteins, and it is strongly expressed in the thalamus during development (Rétaux et al., 1999; Nakagawa 
and O'Leary, 2001). Severe thalamocortical pathfinding defects have been described in Lhx2-null mice (Lakhina et al., 2007), implicating this transcription factor in the guidance of these axons. As $\operatorname{Lh} \times 2$ is also expressed in other forebrain areas, it is essential to restrict the loss of $\operatorname{Lh} x 2$ to thalamic neurons to precisely determine the role of this transcription factor in thalamocortical development.

In the present study we used several in vivo approaches to investigate the influence of $L h \times 2$ on the development of thalamocortical-projecting neurons and the guidance of their axons. Whereas rostral and intermediate thalamic nuclei only weakly express Lhx2 during axon elongation, neurons in the caudal thalamic nuclei, such as the medial geniculate nucleus, retain high levels of Lhx2 during axon projection. Interference with Lhx2 activity in rostrointermediate neurons results in axonal pathfinding defects in vivo, suggesting that the tight regulation of this transcription factor is essential for correct guidance. We identified the guidance receptors Robo1 and Robo2 as downstream targets of the Lhx2 activity that modulates thalamocortical axon (TCA) pathfinding. Together, these findings suggest a model in which the differential expression of Lhx2 in distinct pools of thalamic neurons modulates the expression levels of guidance receptors like Robo1 and Robo2, thereby influencing the rostrocaudal guidance of TCAs in vivo.

\section{Materials and Methods}

Mouse lines. The Gbx2Cre ${ }^{E R}$ line was provided by James Y. Li (University of Connecticut Health Center, Farmington, CT). All mutant mice used for our study were heterozygous for $G b \times 2 C r e^{E R /+}$ and, as expected, they exhibited a normal phenotype (Chen et al., 2009). This line expressed Cre-ires-Egfp under the control of Gbx2 promoter. No homozygotes were used in this study. An $L h x 2^{f l o x /+}$ mouse line was generated and provided by Leif Carlsson (Umeå University, Umeå, Sweden). In these mice, exon 2 of the Lhx 2 locus was flanked by loxP sites (Törnqvist et al., 2010). By crossing the $L h x 2^{f l o x /+}$ mice with the Gbx2Cre $e^{E R /+}$ line, we generated a new transgenic Gbx2Cre $e^{E R /+}: L h x 2^{\text {flox/+ }}$ line, which was in turn crossed with $L h x 2^{\text {flox/+ }}$ females to generate embryos carrying double $L h \times 2$ floxed alleles. Tamoxifen administration generated $G b \times 2 C r e^{E R /+}$ : Lhx $2^{\text {flox/flox }}$ (thalamic deletion of Lhx2; named Th-Lhx2) conditional knock-out mice, carrying a recombinant Lhx2 locus in the Creexpressing cells, and lacking functional Lhx2 in the diencephalic thalamic neuroepithelium (see Results). Lhx2-null mutant embryos (Lhx2 ${ }^{-\prime-}$ ) (Porter et al., 1997; Lakhina et al., 2007) were provided by Shubha Tole (Tata Institute of Fundamental Research, Mumbai, India). Lhx $2^{-\prime-}$ embryos die in utero by embryonic day 15.5 (E15.5), limiting the ages that could be analyzed.

Mouse breeding and genotyping. Mice were maintained on a CD1 genetic background. Noon on the day on which the vaginal plug was found was designated as E0.5. All animal procedures were performed according to the protocols approved by the Committee on Animal Research at the University Miguel Hernández, according to Spanish and European Union regulations. Mouse genotyping was performed by PCR and the following primers were used to determine the expression of the Gbx2Cre $e^{E R}$ transgene: Gbx2: forward, GATATCTCACGTACTGACGG, and reverse, TGACCAGAGTCATCCTTAGC; and the floxed Lhx2 allele: loxp1, GCCAGACTAGCAGACGCTGC; sld2, CCACCGGTACTCCTCT TCAGAG. Two doses of $6 \mathrm{mg}$ of tamoxifen (Sigma-Aldrich) in corn oil (final concentration of $30 \mathrm{mg} / \mathrm{ml}$ ) were administered consecutively by oral gavage to pregnant females at E10.5 and E11.5.

In situ hybridization and immunohistochemistry. For in situ hybridization, mouse brains were fixed overnight in 4\% PFA in PBS and $20 \mu \mathrm{m}$ cryostat sections were hybridized with digoxigenin-labeled probes as described previously (López-Bendito et al., 2006). The following cDNA probes were used: Robo1, Robo2, Robo3, Lhx2, Ngn2, and Gbx2. For immunohistochemistry, cultured slices/explants and mouse embryos were fixed in $4 \%$ PFA for $30 \mathrm{~min}$ or $2-3 \mathrm{~h}$, respectively, washed in PBS, blocked with serum and incubated overnight with the primary antibodies followed by the appropriate secondary antibodies. For BrdU triple staining, sections were first processed for Lhx2 and L1 immunohistochemistry, fixed in $4 \%$ PFA for $50 \mathrm{~min}$ and then processed for BrdU staining. Fluorescent slices were counterstained with DAPI (Sigma-Aldrich) to be able to define the cortical compartments. The following primary and secondary antibodies were used here: rat anti-L1 (1/100, Millipore); goat antiLhx2 (1/50, Santa Cruz Biotechnology); mouse anti-BrdU (1/500, Sigma-Aldrich); rabbit anti-GFP (1/1000, Invitrogen); chicken anti-GFP (1/3000, Aves Labs); donkey anti-goat Alexa-488 (1/500, Invitrogen); donkey anti-goat Alexa-546 (1/500, Invitrogen); goat anti-mouse Alexa546 (1/500, Invitrogen); donkey anti-rabbit Alexa-488 (1/500, Invitrogen); goat anti-chicken Alexa-488 (1/500, Invitrogen); donkey anti-rat Alexa-594 (1/500, Invitrogen); rabbit Fab anti-goat Alexa-633 (1/500, Jackson Bioresearch).

Quantification of Lhx2 gradient was done by measuring the fluorescence along a lateromedial line from the ventricular to the pial surface of the thalamus along rostral, intermediate, and caudal levels. The line was normalized by its size throughout the distinct stages and divided into five bins. The fluorescence was normalized to the point of highest fluorescence.

Dye-tracing studies. For axon tracing, embryonic brains were fixed in $4 \%$ PFA overnight. Small DiI (1, '1'-dioctadecyl $3,3,3^{\prime}, 3^{\prime}$ tetramethylindocarbocyanine perchlorate; Invitrogen) or DiA (4-[4(dihexadecylamino) styryl]-N-methylpyridinium iodide) crystals were inserted into the cortex or thalamus and allowed to diffuse at $37^{\circ} \mathrm{C}$. Vibratome sections $(100 \mu \mathrm{m})$ were obtained and counterstained with fluorescent nuclear DAPI dye (Sigma-Aldrich). For dye tracing together with immunohistochemistry, DiI crystal was injected into different cortical areas at E15.5 and E17.5 brains. After diffusion, brains were fixed during $2 \mathrm{~h}$ at room temperature and subsequently sliced at a thickness of $60 \mu \mathrm{m}$ with a Vibratome. Brain slices were blocked with 2\% DMSO, 3\% BSA, $1 \mathrm{mg} / \mathrm{ml}$ digitonin (Sigma-Aldrich) in PBS for $5 \mathrm{~h}$. Primary antibody was incubated in blocking solution overnight at $4^{\circ} \mathrm{C}$. Secondary antibody was incubated in $0.5 \mathrm{mg} / \mathrm{ml}$ digitonin in PBS overnight at $4^{\circ} \mathrm{C}$.

In utero electroporation and axon quantification. Pregnant females were deeply anesthetized with isofluorane at E12.5 and after laparotomy, the embryos were visualized through the uterus with a fiber optic light source. The cDNA encoding Lhx2 (Invitrogen) or Robo1 (Genentech) were subcloned into the $p C A G G$-ires-Egfp plasmid and electroporated in utero (at a concentration of $1.5 \mu \mathrm{g} / \mu \mathrm{l}$ ) along with the Egfp-encoding plasmid $(0.9 \mu \mathrm{g} / \mu \mathrm{l})$ and fast green (1\%, Sigma-Aldrich). An Egfpencoding plasmid was used as control at a concentration of $1.2 \mu \mathrm{g} / \mu \mathrm{l}$. The DNA mix was injected into the third brain ventricle of each embryo through a glass capillary. Negative and positive electrodes were placed near the head of the embryo, and five square electric pulses of $35 \mathrm{~V}$ and 50 $\mathrm{ms}$ were delivered through the uterus at intervals of $950 \mathrm{~ms}$, using a square pulse electroporator CUY21 Edit (NepaGene). The surgical incision was then closed and the embryos were allowed to develop until E17.5, at which point they were recovered, fixed in 4\% PFA, and analyzed. To quantify the trajectory of electroporated thalamocortical axons after in utero electroporation, a line was drawn from the level of the telencephalic-diencephalic boundary to the edge of the third ventricle at two hypothalamic levels (rostral and caudal) (see Fig. 3, schema). The fluorescence of GFP-labeled axons that crossed this line in each of the hypothalamic regions was measured using ImageJ analysis software. The plotted ratio was calculated by dividing the number of pixels along this line that showed a positive fluorescence, after removing the background fluorescence, by the total number of pixels in the line.

In vitro focal electroporation and thalamic explants. Organotypic coronal slice cultures from wild-type E13.5 embryos were established as described previously (López-Bendito et al., 2006). Expression vectors $(1.5-2 \mu \mathrm{g} / \mu \mathrm{l})$ were injected focally and electroporated into the thalamus as described previously (López-Bendito et al., 2006). Gene expression was then analyzed in thalamic explants dissected out from organotypic slices and cultured in Neurobasal medium containing $0.4 \%$ methylcellulose (Sigma-Aldrich) on glass coverslips coated with poly-L-lysine $(10 \%$, Sigma-Aldrich) and laminin (20 $\mu \mathrm{g} / \mathrm{ml}$, Sigma-Aldrich).

Quantitative reverse transcription-PCR. Total RNA from electroporated thalamic explants cultured for $48 \mathrm{~h}$ was extracted with the RNeasy 
Kit (Qiagen). RNA (300 ng) was treated with RNase-free DNaseI (Fermentas) for $30 \mathrm{~min}$ at $37^{\circ} \mathrm{C}$ and then reverse transcribed into singlestranded cDNA for $1 \mathrm{~h}$ at $42^{\circ} \mathrm{C}$ using SuperScript II Reverse Transcriptase and Oligo(dT) ${ }_{12-18}$ primers (Invitrogen). Quantitative real-time PCR was performed on an Applied Biosystems 7300 real-time PCR unit using the Power SYBR Green PCR Master Mix (Applied Biosystems), the cDNAs generated $(5 \mu \mathrm{l})$ and the appropriate primers. The following primer sequences were used: Lhx2: forward ATGCAAGCTCAACCTG GAGT and reverse TGTGCATGTGAAGCAGTTGA; Robol: forward AGGGAAGCCTACGCAGATG and reverse TGGACAGTGGGCGATT TTAT; Robo2: forward GAGAATCGGGTGGGAAAAGT and reverse CA CAAACTGTGGAGGAGCAA; and GAPDH: forward CGGTGCTGAG TATGTCGTGGAGT and reverse CGTGGTTCACACCCATCACAAA. Each independent sample was assayed in duplicate and gene expression was normalized to that of GAPDH.

Phylogenetic footprinting analysis. In silico analysis of the predicted $5^{\prime}$ flanking region of the Mus musculus Robo1, Robo2, or Robo3 (Rig-1) locus (accession numbers NM_019413, NM_175549, and NM_001164767.1, respectively) was performed using the Evolutionary Conserved Regions and the University of California Santa Cruz genome browsers. Putative Lhx2 binding sites match $6 \mathrm{nt}$ of the described Lhx2 consensus sequence $5^{\prime}$ TAATTA-3' (Wilson et al., 2008). The sites selected for the study showed at least $80 \%$ conservation in several species (rat, mouse, dog, chimpanzee, and human).

Chromatin immunoprecipitation assay. A chromatin immunoprecipitation (ChIP) assay was performed using the EZ ChIP Kit (Millipore) and following the manufacturer's instructions. Briefly, mouse E12.5 thalamic cells were cross-linked with $1 \%$ PFA for $10 \mathrm{~min}$ at room temperature and the cells were sonicated in SDS lysis buffer on ice (Bioruptor, Diagenode; $200 \mathrm{~W}$ potency; $40 \mathrm{~s}$ on, $20 \mathrm{~s}$ off; $4 \times 5 \mathrm{~min}$ ), generating soluble chromatin fragments of $100-400 \mathrm{bp}$. Chromatin was immunoprecipitated with goat anti-Lhx2 (5 $\mu \mathrm{g}$, Santa Cruz Biotechnology), mouse anti-RNA polymerase II (positive control) and goat anti-IgG (negative control) antibodies. The DNA sequences immunoprecipitated were then analyzed by PCR using primer pairs spanning Robo1-region2 and Robo1-region1, as well as Robo2-region1, Robo2-region2, Robo2-region3, Robo2-region4, and Robo3-region 1 . The PCR products were sequenced and analyzed by electrophoresis on $2 \%$ agarose gels.

\section{Results}

Expression of Lhx2 protein in the developing thalamus

Lhx2 mRNA is expressed in the thalamus from the onset of thalamic neurogenesis (Nakagawa and O'Leary, 2001; Lakhina et al., 2007). In Lhx2-null mice, severe defects in thalamocortical axon guidance have developed by E14.5 (Lakhina et al., 2007). However, it remains unclear whether Lhx2 exerts this effect in a cellautonomous manner and, moreover, whether it selectively regulates axon guidance in distinct subsets of thalamic neurons in vivo. Because the expression of Lhx2 protein in early developmental stages has yet to be described, we sought to determine the spatiotemporal regulation of the Lhx2 protein in the thalamus when TCAs begin to grow toward the cortex (Fig. 1).

Neurons expressing Lhx2 were observed along the entire rostrocaudal thalamic axis at all embryonic stages studied (E12.5E15.5). Like other members of the LIM-HD transcription factor family (Bach, 2000; Nakagawa and O'Leary, 2001), Lhx2 was primarily expressed by cells outside the proliferative areas, strongly suggesting that it is found in postmitotic neurons (Fig. 1). Interestingly, the mediolateral pattern of Lhx2 expression at the three defined rostrocaudal levels suggested it was regulated dynamically during embryonic development. Thus, a gradient of Lhx2 expression was observed at the rostral and intermediate thalamic levels (rTh and iTh, respectively). While a strong band of Lhx2expressing cells was located just outside the ventricular zone, neurons projecting TCAs (labeled by L1 immunohistochemistry) were mainly located lateral to this region and they only weakly expressed this LIM protein (Fig. $\left.1 B, G-H^{\prime \prime}\right)$. This pattern of expression closely reproduces that described for $L h \times 2$ mRNA, whereby Lhx2 transcripts are expressed robustly in rostromedial nuclei and they are mainly absent from putative ventroposterior (VP) and dorsolateral geniculate (dLG) nuclei (Nakagawa and O'Leary, 2001). Interestingly, while the mediolateral gradient for Lhx2 is also present at caudal thalamic (cTh) structures, a distinct feature at this level is that neurons located in the most lateral part of the thalamus express high levels of Lhx2 during TCA extension at all the developmental stages studied (Fig. 1C, F, I', I'). Due to their caudolateral localization, these neurons are likely to be part of the putative medial geniculate (MGv) nucleus responsible for relaying auditory information. This rostrocaudal and mediolateral dynamic regulation of $\operatorname{Lhx} 2$ (Fig. $1 \mathrm{~J}, \mathrm{~K}$ ) indicates that there are distinct pools of thalamocortical-projecting neurons dynamically expressing this protein during axonal pathfinding.

\section{Postmitotic $\mathrm{Lhx} 2$ is dynamically regulated in specific subsets of thalamocortical neurons}

Our findings demonstrate that the expression of $\operatorname{Lhx} 2$ protein is developmentally regulated in thalamic neurons. Indeed, while rTh and iTh neurons express Lhx2 weakly during axonal projection, it is strongly expressed by specific populations of cTh neurons. As Lhx2 is expressed by a group of cells that form a band outside the ventricular zone at the stages studied, it is possible that Lhx2 is transiently expressed by all thalamic neurons and then specifically downregulated in distinct subsets of neurons at later stages. To explore this possibility, we followed a single cohort of thalamic neurons by labeling cells born at E12.5 and E14.5 with a BrdU pulse, and analyzing their rostrocaudal distribution and colocalization with Lhx2 and L1 after several hours (Fig. 2). As expected, at both developmental stages, Lhx2 expression appeared to be dynamically regulated in thalamic neurons along both the lateromedial and rostrocaudal axes. Lhx2 expression was activated $\sim 16-18 \mathrm{~h}$ after the BrdU pulse and it was detected in cells located medially, outside of the progenitor domains (Fig. $2 A-D)$. At this time, a high percentage of BrdU-positive cells were found to express Lhx2 at all rostrocaudal levels (BrdU+/ Lhx2+ at E12.5: rTh, 73\%; iTh, 92\%; cTh, 95\%; Fig. $2 H$ ). These Lhx2/BrdU-positive cells were not observed in regions containing L1-positive fibers, suggesting that high Lhx2 expression occurs before these neurons project axons. After $48 \mathrm{~h}, \mathrm{BrdU}-$ positive cells in both the rTh and iTh expressed very low levels of Lhx2 (BrdU+/Lhx2+ at E12.5: rTh, 8\%; iTh, 0\%; Fig. 2I), and occupied more lateral positions colocalizing with L1-positive TCAs (Fig. $2 E, F$ ). The strong colocalization of Lhx2 and BrdU in rTh and iTh lasted only a few hours, since many BrdU-positive cells only weakly expressed Lhx 2 protein $20 \mathrm{~h}$ post-pulse (data not shown). Interestingly, $\sim 98 \%$ of BrdU + cells at the cTh level that moved to lateral positions maintained high Lhx2 expression during axonal pathfinding (Fig. 2G, $G^{\prime}, I$ ).

To determine the specific subset of $\mathrm{rTh}$, iTh, and cTh neurons that dynamically regulate $\mathrm{Lhx} 2$, we combined retrograde DiI tracing from principal cortical areas with immunohistochemistry for Lhx2 at E15.5, when interconnectivity between cortex and thalamus is established (López-Bendito and Molnár, 2003). After DiI injections in primary motor (M1) or somatosensory (S1) areas, only a very weak expression of Lhx 2 was found in DiI-backlabeled cells located in the corresponding thalamic nuclei, which are, respectively, the ventrolateral (VL) nucleus and the ventrobasal (VB) nucleus (Fig. $2 J-K$ '). No detectable expression of Lhx2 was observed in retrograde-labeled thalamic neurons in the dLG 


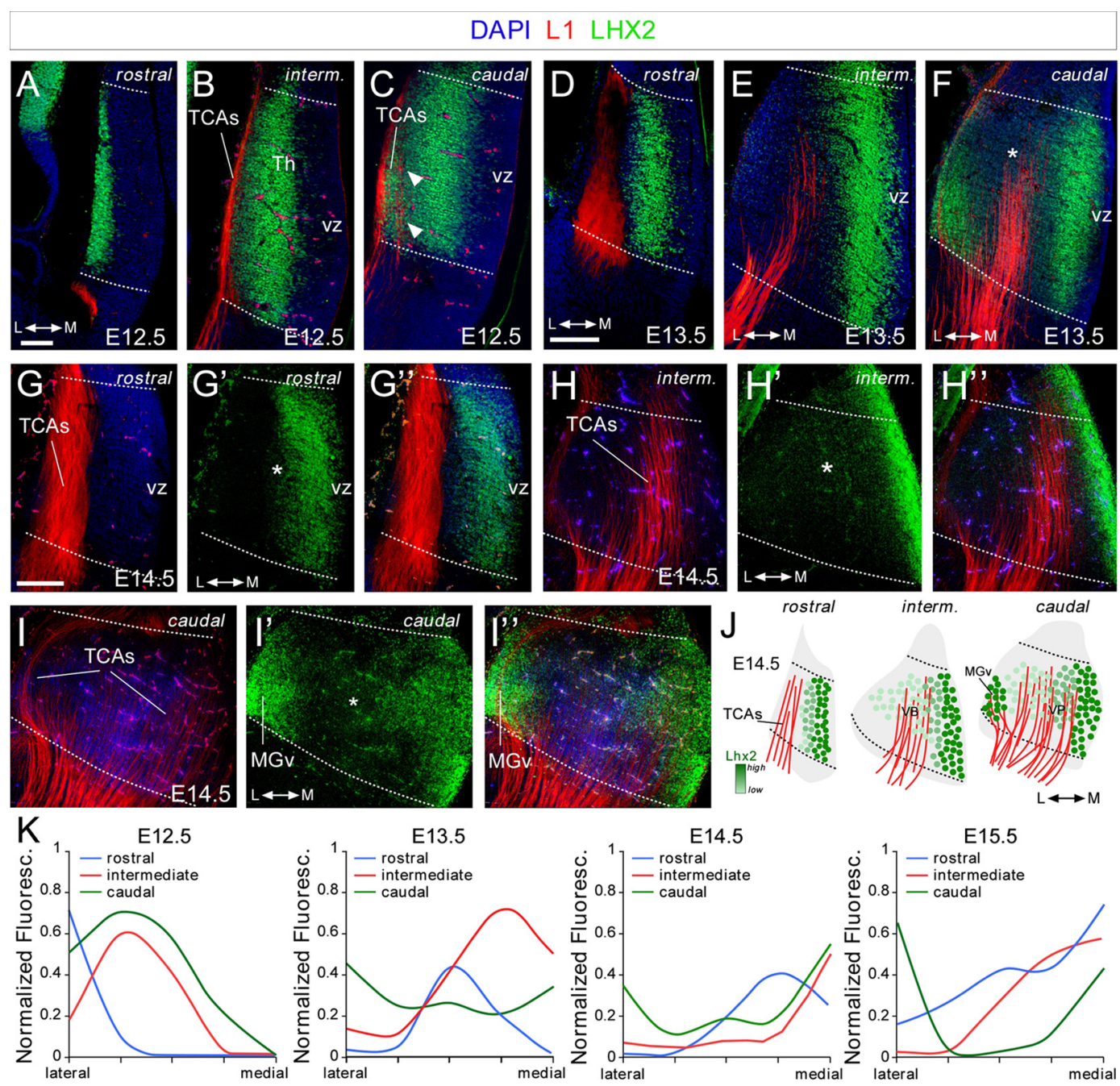

Figure 1. Lhx2 protein expression in the forebrain during development. $\boldsymbol{A}-\boldsymbol{I}^{\prime \prime}$, Coronal sections from rostral, intermediate, and caudal levels of E12.5 (A-C), E13.5 (D-F), and E14.5 $\left(\mathbf{G}-\boldsymbol{I}^{\prime}\right)$ embryonic brains showing Lhx2 (green) and L1 (red) immunohistochemistry. Nuclear DAPI staining is shown in blue. Arrowheads indicate Lhx2-positive cells that emit L1 thalamocortical axons at caudal levels. Dotted lines define the border of the thalamus. Rostral $\left(\mathbf{G}-\boldsymbol{G}^{\prime}\right)$, intermediate $\left(\boldsymbol{H}-\boldsymbol{H}^{\prime}\right)$, and caudal $\left(\boldsymbol{I}-\boldsymbol{I}^{\prime \prime}\right)$ coronal section images of E14.5 embryonic brains showing the spatial relationship between the Lhx2-expressing cells and the L1-positive axons emerging from the thalamus. Asterisks show the Lhx2 gradient in the thalamus. $J$, Diagram of rostrocaudal Lhx2-expressing cells showing the gradient of Lhx2 (light to dark green) as well as L1-positive axons (red) coming out from the thalamus. $\boldsymbol{K}$, Quantification of the Lhx2 expression gradient along the anterior-posterior axes at E12.5, E13.5, E14.5, and E15.5. Th, thalamus; vz, ventricular zone; L, lateral thalamus; M, medial thalamus. Scale bars: (in $\boldsymbol{A}) \boldsymbol{A}-\boldsymbol{C}, 100 \mu \mathrm{m}$; (in $\boldsymbol{D}) \boldsymbol{D}-\boldsymbol{F}, 100 \mu \mathrm{m}$; (in $\mathbf{G}) \mathbf{G}-\boldsymbol{I}^{\prime \prime}, 100 \mu \mathrm{m}$.

nucleus of the thalamus after primary visual area (V1) injections (Fig. $2 L, L$ '). As expected, at cTh levels, only DiI-back-labeled cells at the MGv expressed high levels of Lhx2 at the time of projecting to auditory primary cortical area (A1) (Fig. 2M, $M^{\prime}$ ). Moreover, this pattern of Lhx2 expression in distinct thalamicprojecting neurons was maintained at later embryonic stages (data not shown). In sum, Lhx2 protein was transiently expressed by a high percentage of early postmitotic thalamic neurons $(\sim 16-18$ h; Fig. $2 N)$ and was quickly downregulated in $\mathrm{rTh}$ (as $\mathrm{VL})$ and iTh (as VB) neurons during axonal outgrowth $(\sim 48 \mathrm{~h}$, Fig. $2 N$ ). By contrast, cTh neurons, as MGv thalamocorticalprojecting neurons, retained high levels of Lhx2 during axonal pathfinding (Fig. $2 N$ ). This dynamic regulation of Lhx2 appears to occur in cycles during thalamic neurogenesis, as was observed repeatedly at distinct developmental stages (Fig. 1; data not shown). These experiments suggest that Lhx2 expression is tightly regulated in distinct populations of thalamocortical neurons.

\section{Overexpression of Lhx 2 causes thalamocortical axon} guidance defects in vivo

Our observations raised the intriguing possibility that it may be necessary to downregulate Lhx2 protein in rTh and iTh neurons for them to acquire thalamocortical guidance properties. Accordingly, strong Lhx2 expression in rTh and iTh neurons during axonal outgrowth may give rise to axonal pathfinding defects. To test this hypothesis, we induced $L h x 2$ expression in thalamic progenitors through in utero electroporation at E12.5 and compared the distribution and localization of Egfp-labeled axons at E17.5 (Fig. 3A,B). At this time, neurogenesis has largely ceased in the cTh nuclei, including the MGv, and the generation of rTh and iTh precursors has begun (Angevine, 1970). Thus, electroporation at E12.5 primarily targets rTh and iTh neurons (Fig. $3 C$ ). As expected, double immunohistochemistry against Lhx2 and Gfp revealed strong ectopic Lhx2 protein expression in iTh neurons, including those of the VP and dLG, when compared with the non-electroporated control hemisphere (Fig. 3D-F'). In control experiments, axons expressing Gfp 
DAPI Lhx2 L1 BrdU
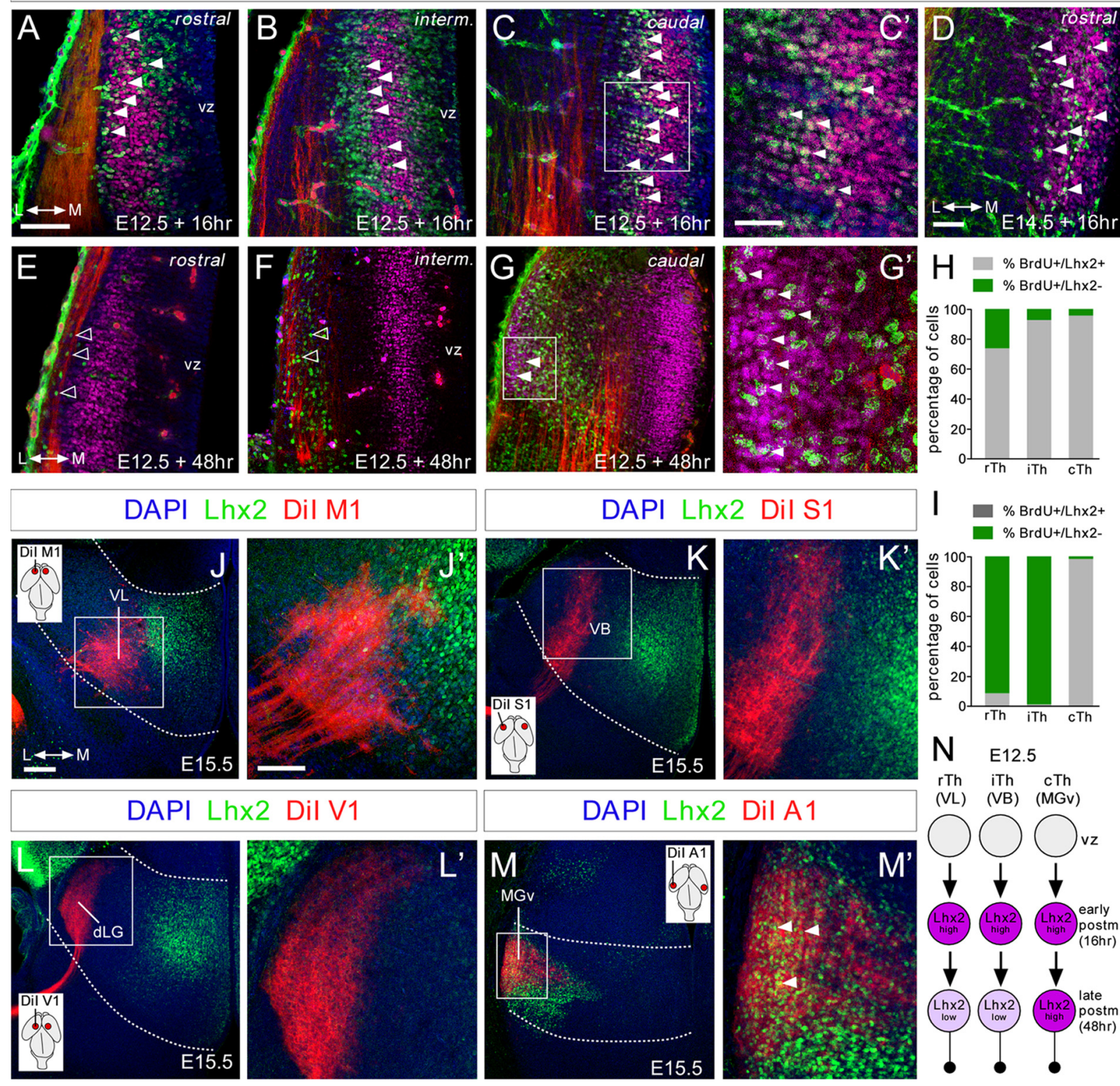

rTh iTh CTh

(VL) (VB) (MGv)
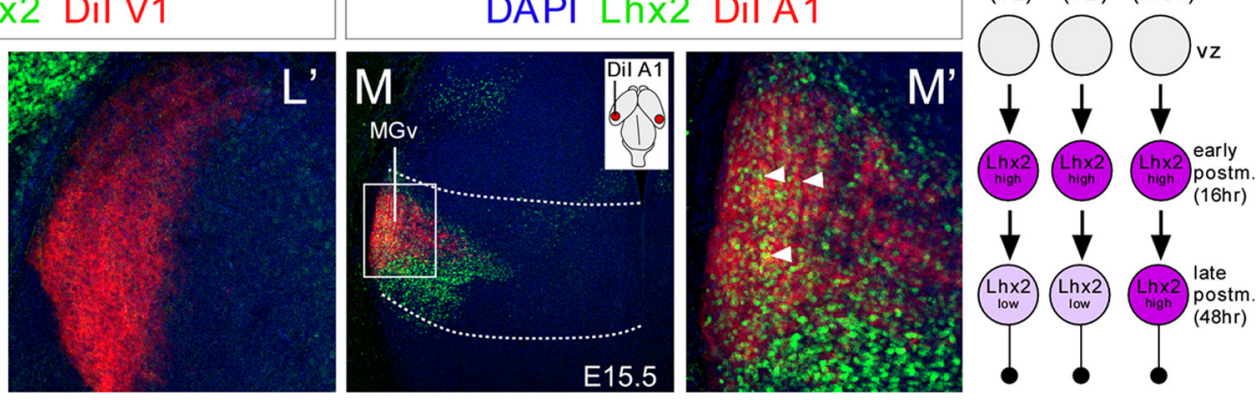

Figure 2. Lhx2 expression is dynamically regulated in postmitotic thalamic neurons. $A-G^{\prime}$, Coronal sections showing immunohistochemistry for Lhx2 (purple), L1 (red), and BrdU (green), after BrdU was injected at E12.5 and E14.5 (D) and allowed to incorporate for $16(\boldsymbol{A}-\boldsymbol{D})$ or $48 \mathrm{~h}(\boldsymbol{E}-\boldsymbol{G})$. Nuclear DAPI staining is shown in blue. A large percentage of cells at rostral levels and nearly all at intermediate and caudal levels that incorporated BrdU after a $16 \mathrm{~h}$ pulse strongly expressed Lhx2 atE12.5 (A-C', filled arrowheads). D, The majority of the cells that incorporated BrdU after a $16 \mathrm{~h}$ pulse strongly express $L \mathrm{hx} 2$ at E14.5. After a $48 \mathrm{~h}$ pulse, cells that incorporated BrdU at rostral and intermediate levels showed a strong reduction in Lhx2 expression ( $\boldsymbol{E}, \boldsymbol{F}$, open arrowheads), although it remained high in caudolateral regions ( $\mathbf{G}-\boldsymbol{G}^{\prime}$, filled arrowheads). $\boldsymbol{C}^{\prime}, \boldsymbol{G}^{\prime}$, High-magnification images showing colocalization (white) of Lhx2-positive (purple) and BrdU-positive (green) cells with projecting axons (red) at caudal thalamic levels after a 16 and $48 \mathrm{~h}$ pulse, respectively. $\boldsymbol{H}, \boldsymbol{I}$, Quantification of the percentage of BrdU +/Lhx2 + cells (gray bars) and BrdU +/Lhx2 - cells (green bars) along the rostrocaudal axes at E12.5, after 16 and $48 \mathrm{~h}$ BrdU injection, respectively. $J-M^{\prime}$, Coronal sections of Dil-injected brains (red) into M1 $(J, J), S 1(K, K), V 1(L, L)$, and A1 $(M, M)$ cortical areas, showing retrograde-labeled cells in the VL, VB, dLG, and MGv thalamic nuclei, respectively.Immunohistochemistry showing Lhx2 expression pattern (green) at the different thalamicnuclei and its relation with the back-labeled cells from the different cortical areas. Only back-labeled axonal fibers from the A1 cortex showed a strong colocalization with Lhx2-positive cells at caudal thalamic levels ( $M^{\prime}$, arrowheads). Insets show the cortical area in which the Dil crystal was placed. $N$, Diagram showing the dynamic regulation of $L h x 2$ protein in thalamocortical neurons. vz, ventricular zone. Scale bars: (in $A) A, B, C, E, F, G, 100 \mu \mathrm{m}$; (in $\left.C^{\prime}\right) C^{\prime}, D, G^{\prime}, 40 \mu \mathrm{m}$; (in J) J, $K, L, M, 100 \mu \mathrm{m}$; (in $\left.J\right) J^{\prime}, K^{\prime}, L^{\prime}, M^{\prime}, 50 \mu \mathrm{m}$.

extended dorsally through the diencephalon and crossed the diencephalic-telencephalic boundary, following their normal route toward the cortex. A minority of these axons invaded the hypothalamic region $(r=0.33 \pm 0.04, n=10$ embryos; Fig. $3 H, H$,J). In contrast, in embryos electroporated with $L h x 2$, a significant proportion of thalamic axons accumulated in the hypothalamus at E17.5 ( $r=0.58 \pm 0.02, n=9$ embryos; Fig. $\left.3 J-K^{\prime}\right)$. Consequently, fewer Gfp-expressing axons were observed extending through the cortical wall when $L h \times 2$ was overexpressed (Fig. 3I,L). Together these results demonstrate that interfering with the dynamics of Lhx2 expression in thalamic neurons produces erroneous axonal guidance in vivo, suggesting that specific developmental programs are likely to precisely regulate the expression of this factor.

\section{Lhx 2 represses Robo1 and Robo 2 mRNA expression in thalamocortical neurons}

The findings above demonstrate that overexpression of Lhx2 expression in rTh and iTh neurons leads to TCA invasion of inappropriate areas in the hypothalamus, diminishing the number of 

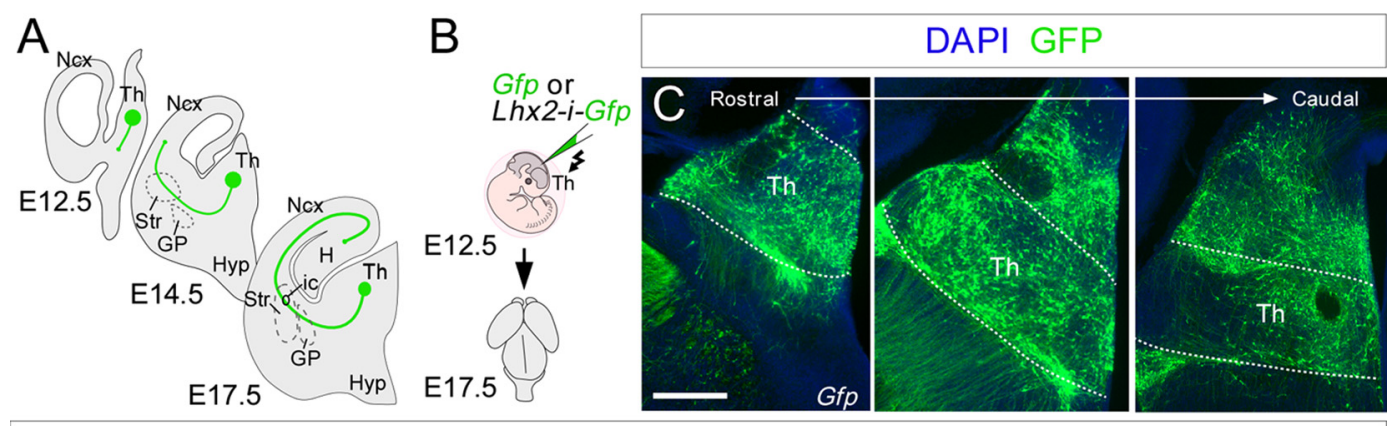

DAPI LHX2 GFP
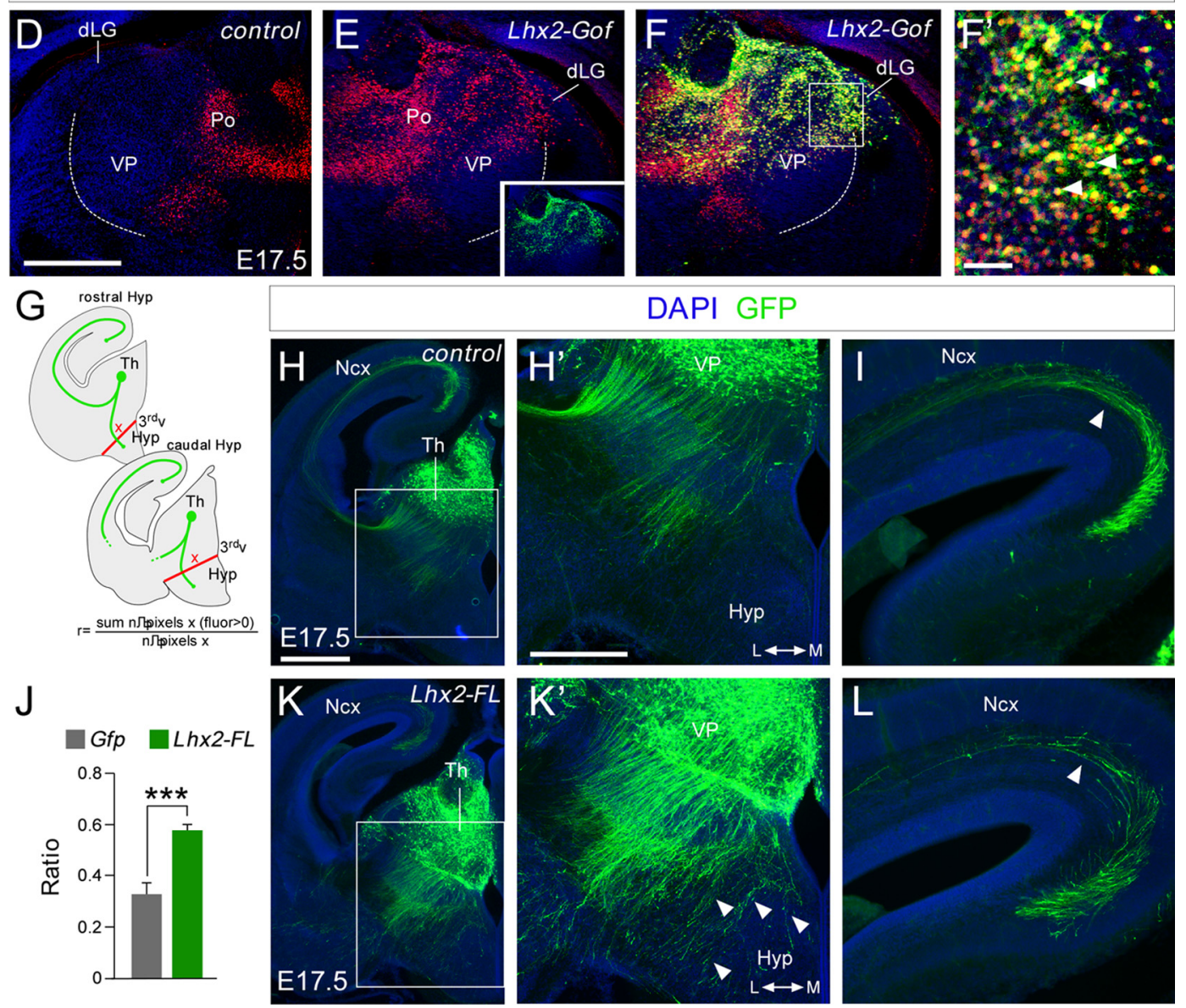

Figure 3. Lhx2 overexpression in the thalamus produces defects in thalamocortical guidance. $A$, Diagram illustrating how thalamocortical axons travel from the thalamus to the cortex at different embryonic stages. B, Schematic diagram of the experimental design used. C, Example of electroporated brain, showing the GFP expression in coronal sections at rostral, intermediate, and caudal thalamic levels respectively. $\boldsymbol{D}-\boldsymbol{F}^{\prime}, \mathrm{Lhx} 2$ immunohistochemistry in control ( $\boldsymbol{D}$ ) and Lhx2-electroporated $(\boldsymbol{E})$ hemispheres from the same embryo at E17.5. Note $L$ hx2 is ectopically overexpressed in the electroporated side $(\boldsymbol{E})$. We inverted $\boldsymbol{D}$ for a better comparison between the nonelectroporated hemisphere $(\boldsymbol{D})$ and the electroporated one $(\boldsymbol{E}-\boldsymbol{F})$. The inset in $\boldsymbol{E}$ indicates the extension of the electroporated area. $\mathbf{G}$, Diagram of the quantification performed. $\boldsymbol{H}-\boldsymbol{L}$, Coronal sections of E17.5 brains showing GFP (green) immunohistochemistry after in utero electroporation at E12.5 with Egfp or Lhx2-ires-Egfp-expressing plasmids. Nuclear DAPI staining is shown in blue. $\boldsymbol{H}^{\prime}, \boldsymbol{K}^{\prime}$, Higher-magnification views of $\boldsymbol{H}$ and $\boldsymbol{K}$ to highlight the thalamocortical guidance defects after Lhx2 overexpression. Derailed thalamocortical axons invade the hypothalamic region (arrowheads) in brains overexpressing Lhx2 but not in control brains. $I, L$, Fewer electroporated axons reach the neocortex when Lhx2 is upregulated in rTh and iTh neurons (arrowheads). J, Quantification of the derailment of TCAs at the hypothalamus. The ratio is the number of pixels along the red lines showing a positive fluorescence after removing the background fluorescence, divided by the total number of pixels along the red lines. Asterisks indicate significance at ${ }^{* * *} p<0.001$; Student's $t$ test. Data are presented as the mean \pm SEM. Th, thalamus; Ncx, neocortex; Str, striatum; GP, globus palidus; Hyp, hypothalamus; H, hypocampus; $3^{\text {rd }} v$, third ventricle; Po, posterior group of thalamic nuclei. Scale bars: (in $\boldsymbol{H}$ ) $\boldsymbol{H}, \boldsymbol{K}, 200 \mu \mathrm{m}$; (in $\boldsymbol{C}) \boldsymbol{C}, \boldsymbol{H}^{\prime}, \boldsymbol{I}, \boldsymbol{K}^{\prime}, \boldsymbol{L}, 100 \mu \mathrm{m}$; (in $\left.\boldsymbol{D}\right) \boldsymbol{D}-\boldsymbol{F}, 100 \mu \mathrm{m} ; \boldsymbol{F}^{\prime}, 50 \mu \mathrm{m}$.

axons that reach the cortex. These defects strongly resemble those observed in the absence of Slit/Robo signaling (Bagri et al., 2002; López-Bendito et al., 2007). Slit proteins, expressed in hypothalamic regions, repel and prevent the entry of TCAs expressing Robo receptors to these tissues. Accordingly, the absence of Robo receptors or Slit ligands provokes the aberrant invasion of the hypothalamus by TCAs, particularly those arising from iTh nu- clei (Bagri et al., 2002; López-Bendito et al., 2007). In situ hybridization for $L h x 2$, Robo1, and Robo2 receptors revealed that Lhx2thalamic neurons are likely to express Robol at early postmitotic stages and at all rostrocaudal levels (Fig. $4 A-F^{\prime}$ ). However, once thalamic neurons begin to differentiate and segregate into their distinct nuclei in the thalamic mantle, they primarily express Robo2 and only weak $\operatorname{Lh} \times 2$ (Fig. 4G-J'). The exceptions are those 

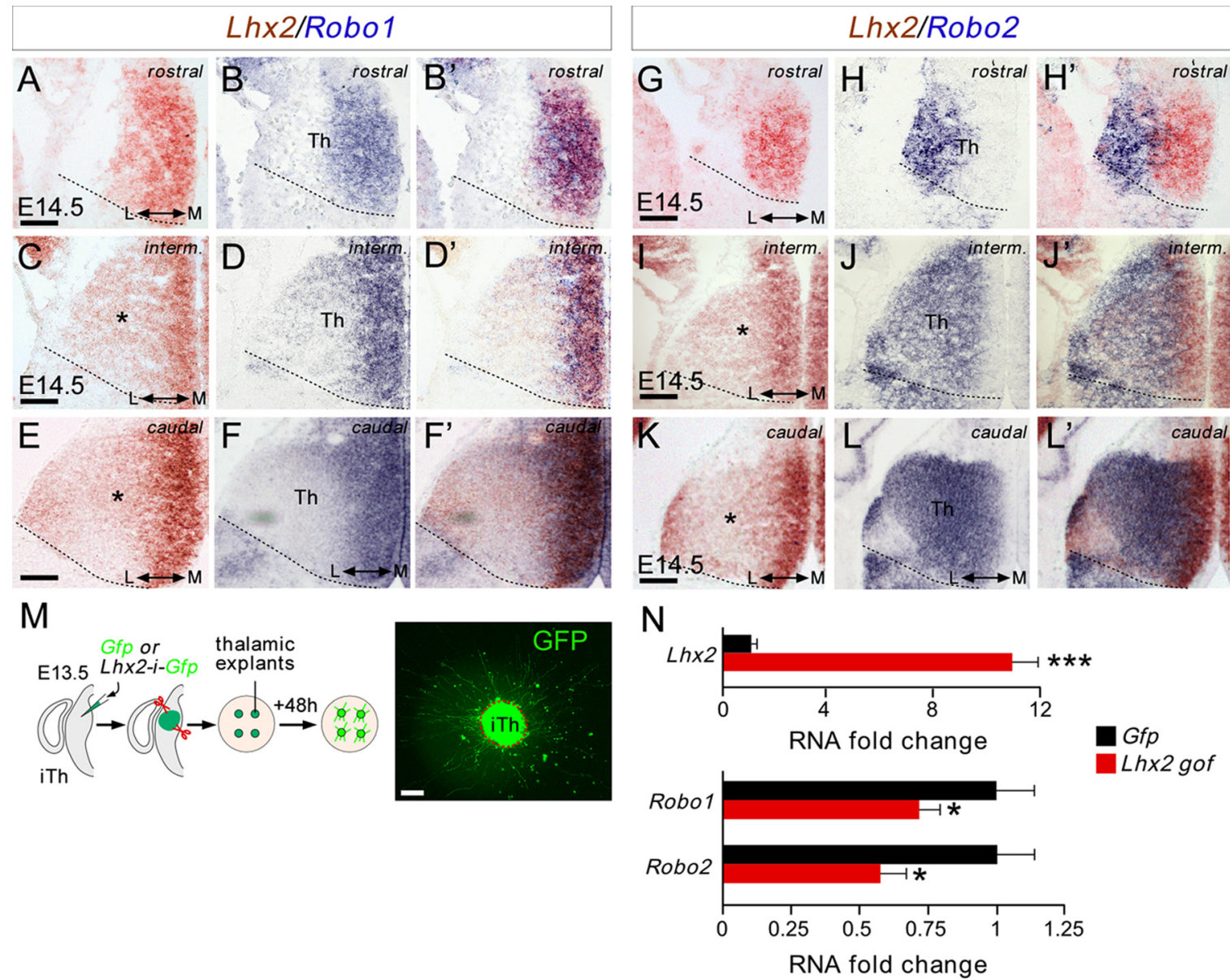

Figure 4. Lhx2 represses Robo1 and Robo2 expression in thalamic-projecting neurons. $A-L^{\prime}$, Consecutive coronal cryostat sections at rostral, intermediate, and caudal thalamic levels of E14.5 wild-type embryos showing $L h \times 2(\boldsymbol{A}, \boldsymbol{C}, \boldsymbol{E}, \boldsymbol{G}, \boldsymbol{I}, \boldsymbol{K}$, red), Robo1 ( $\boldsymbol{B}, \boldsymbol{D}, \boldsymbol{F}$, blue), and $R o b 02(\boldsymbol{H}, J, L$, blue) mRNA expression. The merged images are a composite of two consecutive sections for $L h \times 2$ and Robo1 $\left(\boldsymbol{B}^{\prime}, \boldsymbol{D}^{\prime}, \boldsymbol{F}^{\prime}\right)$ or Robo2 $\left(\boldsymbol{H}^{\prime}, \boldsymbol{J}^{\prime}, \boldsymbol{L}^{\prime}\right)$ in situ hybridization. Asterisks highlight the Lhx2 gradients at the mRNA level. $\boldsymbol{M}$, Schematic diagram of the experimental paradigm used for quantitative real-time PCR assays. E13.5 slices were electroporated focally with Egfp-coding or Lhx2-ires-Egfp-coding plasmids. Thalamic explants were dissected from the electroporated region and allowed to grow on coverslips coated with poly-L-lysine and laminin. $\boldsymbol{N}$, Quantitative real-time PCR analysis of Robo1 and Robo2 expression in Egfp-electroporated and Lhx2-electroporated thalamic cells. Histograms show the fold change in RNA expression for Lhx2. Gene expression was normalized using GAPDH. Control conditions where normalized to 1 ( \pm SEM). Asterisks indicate significance at ${ }^{*} p<0.05$ and ${ }^{* * *} p<0.001$, Student's $t$ test. Scale bars: $A-L^{\prime}, 200 \mu \mathrm{m} ; M, 50 \mu \mathrm{m}$.

in the putative MGv population, which retain high levels of Lhx2 at cTh levels (Fig. $4 K-L^{\prime}$ ). Thus, we hypothesized that Lhx2 may modulate Robo1 and Robo2 receptor expression in thalamicprojecting neurons. Accordingly, low levels of $\operatorname{Lh} \times 2$ in $\mathrm{rTh}$ and iTh neurons would facilitate strong expression of Robo receptors (especially Robo2), which would prevent their axons from invading the hypothalamus. This hypothesis was tested in vitro by electroporating iTh explants with Lhx2-ires-Gfp (or with Gfp plasmid alone) and assessing gene expression in growing axons and neurons after $48 \mathrm{~h}$ in culture (Fig. $4 \mathrm{M}$ ). Quantitative real-time PCR revealed a dramatic increase in the expression of $L h \times 2$ in iThprojecting neurons expressing Lhx2-ires-Gfp compared with those expressing the control Gfp (control $=1 \pm 0.21$, Lhx2 = $10.79 \pm 1.79, n=9$; Fig. $4 N$ ). Remarkably, overexpression of Lh $\times 2$ decreased the transcription of Robol by $28 \%$ (control $=1 \pm$ $0.14, L h \times 2=0.72 \pm 0.08 ; n=12$ ) and that of Robo2 by $42 \%$ (control $=1 \pm 0.18, L h \times 2=0.58 \pm 0.09, n=9$ : Fig. $4 N)$. Interestingly, we found that the expression of Lhx9, another LIM-HD protein expressed in the thalamus (Rétaux et al., 1999), is also downregulated in response to Lhx2 overexpression (data not shown), suggesting that these two members of this family of transcription factors are regulated in the thalamus. Together, these results suggest that Lhx2 may regulate TCA guidance by modulating the expression of Robol and Robo2 receptors.

\section{Lhx2 represses the transcription of Robo 1 and Robo 2 in thalamic cells}

In commissural neurons of the developing spinal cord, Lhx2 has been shown to indirectly influence the function of Robol and Robo2 receptors by regulating Rig-1, as demonstrated by electrophoretic mobility shift assay (EMSA) in nuclear extracts from COS cells (Wilson et al., 2008). However, Robo3 mRNA is not expressed in developing thalamic neurons in either control conditions, or following $L h \times 2$ overexpression (data not shown). Therefore, opposite to the spinal cord, Lhx2 may influence Robo 1 and Robo2 expression in thalamic neurons by direct binding to Robo1- and Robo2-specific regulatory sequences. To investigate this possibility, we performed a phylogenetic footprinting analysis of the regions $20 \mathrm{~kb}$ upstream and $70 \mathrm{~kb}$ downstream of the Robol and Robo2 transcription initiation site. This approach revealed two putative Robo1 (Fig. 5A) and four putative Robo2 (Fig. $5 B$ ) regulatory regions containing Lhx2-binding sequences with evolutionary conserved regions, which we designated as Robol-region1 (from -7674 bp 5'- 
A
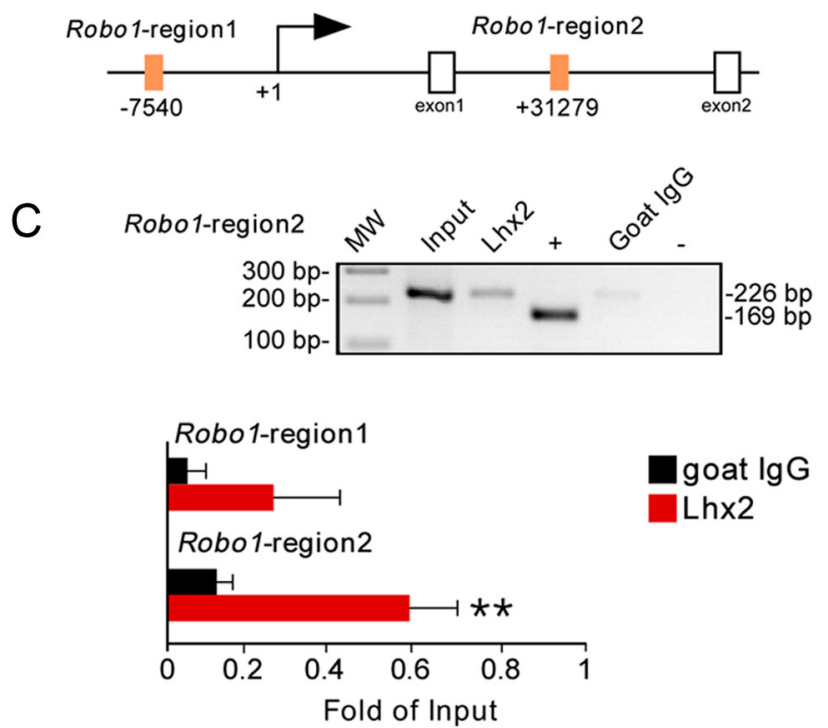

E

\section{Robo1-region2}

RAT CCCCTCTGAATGTATTGATTTTTTAAAATATAATTATACTGTGGATTTAATTTAGTATATGCGT MOUSE CCCCTCCGAATGTATTGATTTTTCAA--TATAATTATAATGTGGATTTAATTTAGTATATGCGT CHIMP TCTTACTTAATGTATTGATTTTTAAA--TATAATTATAACATGAATTTAATTGAATATATGTGT MACAQ TCTTCCTTAATGTATTGATTTTTAAA--TATAATTATAACATGAATTTAATTCAGTATATGTGT HUMAN TCTTACTTAATGTATTGATTTTTAAA--TATAATTATAACATTAATTTAATTGAATATATGTGT CANIS TCTTACTTAATGTATTGATTTTTAAA--TATAATTATAATGTGAATTTAATTTGGTATATGTGTT

G

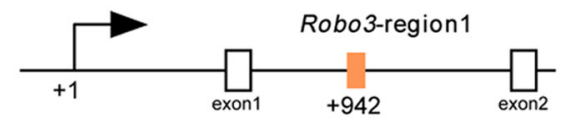

$\mathrm{H}$

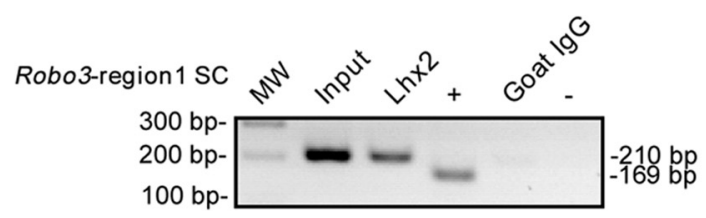

B
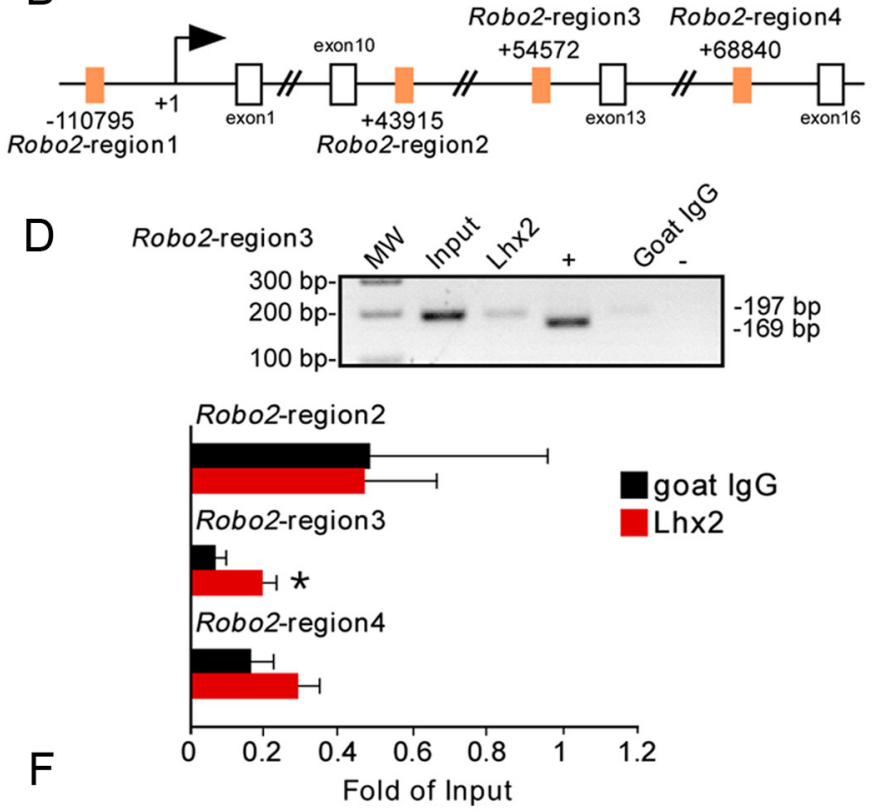

Robo2-region3

RAT AAGAAC-CAAGTACTTTGTTGGAATGAGACGTAATTAGACACTTCAGTTTCAATTGTACTTATTTC' MOUSE AAGAAC-AAAGTAATTTGTTGGAATGAGACTTAATTAGACACATCAGTGACAATCATACCTATT-CC CHIMP AAGAGCAAAACTAATTTGTTGGACTAAAAATTAATTAGACACATCAGATTCGATTGTACCTATTACC MACAO AAGAACAAAACTAATTTGTTGGACTAAAAACTAATTAGACACATCAGGTTTGATTGTACCTATTACC HUMAN AAGAACAAAACTAATTTGTTGGACTAAAAATTAATTAGACACATCAGATTCGATTGTACCTATTACC CANIS AAGAACAAAACTAATTTGTTGGACTAAAAATTAATTAGACACATCAGATTCGATTGTACCTATTACT

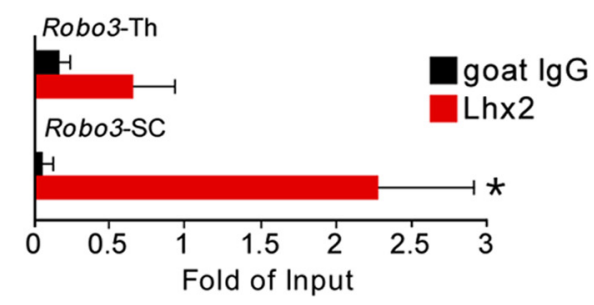

Figure 5. In thalamic neurons, Lhx2 binds to regulatory sequences in the Robo1 and Robo2 genes in vivo. A, B, G, Putative Lhx2 DNA binding sites (orange boxes indicate 6 bp consensus sequences) in the Robo1, Robo2, and Robo3 genes, respectively. $\boldsymbol{C}_{\mathbf{D}} \mathbf{D}, \boldsymbol{H}$, ChIP assays reveal that Lhx2 binds to specific enhancer region (TAATTA) of Robo1, Robo2, and Robo3 (in spinal cord) in the embryonic E12.5 thalamic tissue in vivo. Input chromatin represents $1 \%$ of the total chromatin. Anti-RNA polymerase ll antibody was used as positive control and DNA amplified using control primers specific for the GAPDH promoter. Nonspecific goat serum (goat $\mathrm{lgG}$ ) was used as negative control (IgGs), and DNA fragments were amplified with primers flanking the studied regions of Robo1, Rob02, or Rob03. The amplicon size (number of base pairs) is indicated for each regulatory sequence. The plotted bars indicate the intensity of the PCR bands, for each region, quantified and normalized to their corresponding input band. For Robo1-region1: $0.05 \pm 0.04$ (goat lgG) and $0.26 \pm 0.16$ (Lhx2). For Robo1-region2: $0.12 \pm 0.04$ (goat lgG) and $0.58 \pm 0.12$ (Lhx2). For Robo2-region2: $0.80 \pm 0.71$ (goat lgG) and $0.78 \pm 0.33$ (Lhx2). For Robo2-region3: $0.11 \pm 0.04$ (goat lgG) and $0.32 \pm 0.05$ (Lhx2). For Robo2-region4: $0.27 \pm 0.10$ (goat lgG) and $0.48 \pm 0.10$ (Lhx2). For Rob03 in spinal cord: $0.05 \pm 0.03$ (goat lgG) and $2.28 \pm 0.68$ (Lhx2). For Robo3 in thalamus: $0.17 \pm 0.08$ (goat lgG) and $0.65 \pm 0.28$ (Lhx2). Asterisks indicate significance at ${ }^{*} p<0.05$ and ${ }^{* *} p<0.01$, Student's $t$ test. Data are presented as the mean \pm SEM. E, F, Putative Lhx2-binding sequence of genomic rat, mouse, chimpanzee, macaque, human, and dog in Robo 1 and Robo2, respectively, showing the degree of conservation between these different species.

CGATA-3' to -7432bp 5'-ACAAG-3'), Robo1-region2 (from +31191bp 5'-GGGAG-3' to +31417bp 5'-AGGTG-3'), Robo2region1 (from $-110877 \mathrm{bp} 5^{\prime}$-GGTTC-3' to $-110667 \mathrm{bp} 5^{\prime}-$ GAAGC-3'), Robo2-region2 (from +43823 pb 5'-GCTTC-3' to +44037bp 5'-CAGCC-3'), Robo2-region3 (from +54464bp 5' CCCAC-3' to +54661bp 5'-AAAGG-3'), and Robo2-region4 (from +68703 bp $5^{\prime}$-GGCAT-3' to +68927 bp $5^{\prime}$-ACTGG-3'). ChIP analyses demonstrated that Lhx2 binds to the Robo1region2 in E12.5 thalamic neurons $(n=10)$, but does not significantly bind to the Robol-region 1 ( $n=6$; Fig. $5 C$ ). Analysis of the four regions of interest for the Robo2 gene revealed significant binding of Lhx2 to the Robo2-region3 ( $n=7$; Fig. $5 D$ ). In contrast, Lhx2 appeared not to form a complex with Robo2-region 1 $(n=6$; Fig. $5 D)$, Robo2-region2 $(n=6$; Fig. $5 D)$, or Robo2- region4 ( $n=6$; Fig. $5 D$ ). Furthermore, we confirmed that Lhx2 bound to the Robo3 regulatory region that we designated as Robo3-region1 (from +639bp 5'-GAAAT-3' to +1287bp 5'GCTGC-3'; Wilson et al., 2008) in E12.5 spinal cord extracts ( $n=$ 4 ) but failed to bind to this region in thalamic neurons ( $n=6$; Fig. $5 H)$. Together, these results demonstrate that Lhx2 functions as a transcriptional regulator of Robo1 and Robo2 receptor expression in thalamic neurons.

\section{Conditional deletion of $L h \times 2$ in thalamic neurons}

To further assess how the dynamic regulation of Lhx2 affects the development of thalamocortical neurons in vivo, we designed a strategy to conditionally delete Lhx2 in the thalamus. Accordingly, we bred mice carrying floxed alleles of the $L h \times 2$ locus with 


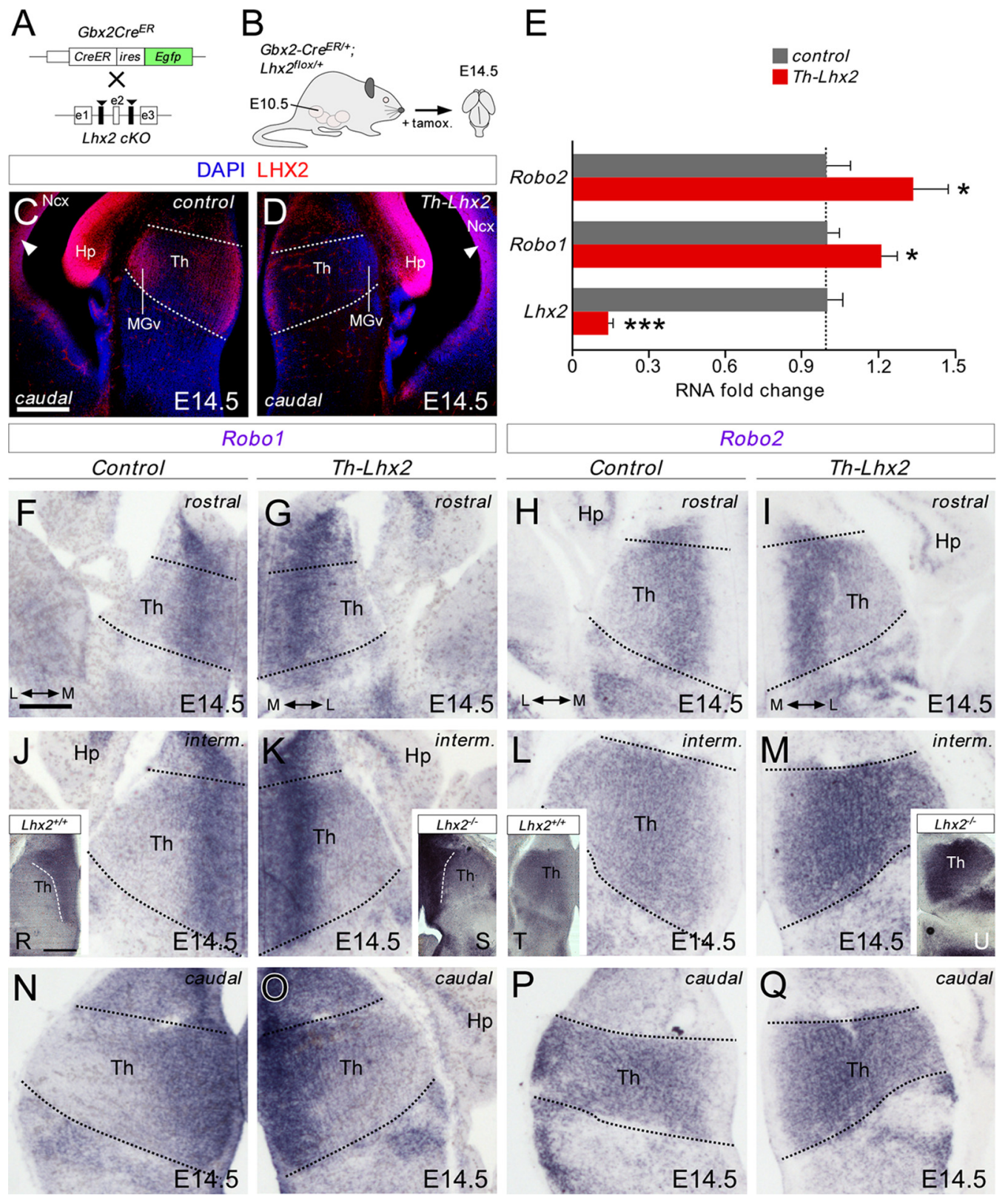

Figure 6. Conditional deletion of $L \mathrm{~h} \times 2$ in thalamic neurons augments Robo 1 and Rob02 receptor expression in the thalamus. $A, B$, Schematic diagram illustrating the strategy used to delete Lhx2 in the thalamus at specific developmental stages. $\boldsymbol{C}, \boldsymbol{D}$, Coronal sections at caudal thalamic levels showing Lhx2 immunohistochemistry in control ( $\boldsymbol{C}$ ) and in conditional thalamic Lhx2 knock-out mice (D, Th-Lhx2) at E14.5. As expected, Lhx2 expression was strongly reduced in the thalamus of these mice, while the gradient of Lhx2 expression at the cortex remained unaltered (arrowheads). $\boldsymbol{E}$, Quantitative PCR was performed in the thalamus of control and Th-Lhx2 embryos at E14.5. As expected, the levels of Lhx2 were reduced very significantly in the Th-Lhx2 thalamic embryos. However, the levels of Robo1 and Robo2 were increased compared with the control thalamus. Asterisks indicate significance at ${ }^{*} p<0.05,{ }^{* * *} p<0.001$, Student's $t$ test. Data are presented as the mean \pm SEM. $\boldsymbol{F - Q}$, Coronal sections at rostral, intermediate, and caudal thalamic levels showing Robo1 and Robo2 in situ hybridization in control $(\boldsymbol{F}, \boldsymbol{J}, \mathbf{N}, \boldsymbol{H}, \boldsymbol{L}, \boldsymbol{P})$ and $T h-L h \times 2(\mathbf{G}, \boldsymbol{K}, \mathbf{O}, \mathbf{I}, \mathbf{M}, \mathbf{Q})$ embryos at E14.5. The expression of both receptors was increased in the absence of $L$ hx2. $\boldsymbol{R}-\boldsymbol{U}$, Coronal sections at the caudal thalamic level showing the Robol and Robo2 expression in wild-type $(\boldsymbol{R}, \boldsymbol{T})$ and Lhx $2^{-/-}(\boldsymbol{S}, \boldsymbol{U})$ embryos at E14.5, respectively. Th, thalamus; Ncx, neocortex; Hp, hippocampus. Scale bars: (in C) C, D, $200 \mu \mathrm{m}$; (in $\left.\boldsymbol{F}\right) \boldsymbol{F}-\boldsymbol{Q}, 100 \mu \mathrm{m} ; \boldsymbol{R}-\boldsymbol{U}, 200 \mu \mathrm{m}$.

a $\mathrm{Gb} \times 2 \mathrm{Cre} e^{E R /+}$ mouse (Chen et al., 2009) in which tamoxifen inducible CreER (T2) recombinase is expressed under the control of the Gbx2 promoter (Fig. 6A). Gbx2 is a homeobox transcription factor expressed almost exclusively in thalamic neurons during forebrain development (Bulfone et al., 1993; Jones and Rubenstein, 2004; Chen et al., 2009). In addition to its localization in the developing thalamus, Lhx2 is strongly expressed in the neocortex, where it is essential for cortical and hippocampal specification (Mangale et al., 2008; Chou et al., 2009). To verify the efficiency and specificity of recombination in thalamic neurons in the conditional knock-out mice, we analyzed the distribution of cells expressing Lhx2 protein in the forebrain of E14.5 control $\left(G b \times 2 C r e^{E R /+} ; L h x^{2+/+}\right)$ and $\operatorname{Lh} \times 2$ thalamic conditional knockout embryos $\left(\mathrm{Gb} \times 2 \mathrm{Cre}{ }^{E R /+} ; \mathrm{Lh} \times 2^{\text {flox/flox }}\right.$; named Th-Lhx2) after tamoxifen administration at E10.5 and E11.5 consecutively (Fig. $6 B$ ). As expected, expression of Lhx2 in the neocortex and hippocampus was not affected in the Th-Lhx2 mouse, while the number of Lhx2-expressing cells throughout the thalamus fell dramatically at all rostrocaudal levels $(n=4$ controls; $n=3$ Th-Lhx2; Fig. 6C,D; data not shown).

Our gain-of-function experiments suggested that Lhx2 represses Robo receptor expression by thalamic neurons. Thus, we 
tested whether conditional deletion of $\operatorname{Lh} \times 2$ in the thalamus altered the expression of Robol and Robo 2 mRNA transcripts at E14.5. As expected, the levels of $L h x 2$ decreased $>85 \%$ in the thalamus of $T h-L h \times 2$ mouse compared with wild type as shown by quantitative PCR (control: $1 \pm 0.06$; Lhx2: $0.15 \pm 0.01 ; n=$ 11-14; Fig. 6E). Moreover, the lack of $L h \times 2$ in $T h-L h \times 2$ embryos significantly increased Robol (control: $1 \pm 0.05$; Robol: $1.22 \pm$ 0.06; $n=14-15$ ) and Robo2 (control: $1 \pm 0.10$; Robo2: $1.35 \pm$ $0.13 ; n=13-14$ ) mRNA levels in thalamic neurons at E14.5 (Fig. $6 E)$. This increase was not detected in other diencephalic structures as the hypothalamic region (control: $1 \pm 0.14 ; \operatorname{Lh} \times 2: 0.78 \pm$ 0.17; control: $1 \pm 0.06$, Robol: $1.02 \pm 0.04$; control: $1 \pm 0.12$, Robo2: $1.07 \pm 0.08 ; n=8-11$; data not shown). The increase in both receptors in Th-Lhx2 thalamic neurons was observed along the entire rostrocaudal axis (Fig. $6 F-Q$ ) and was more prominent in Lhx2-null mutant mice ( $n=4 \operatorname{Lh} x^{2+/+} ; n=4 \operatorname{Lh} \times 2^{-/-}$; Fig. $6 R-U)$. Together these results demonstrate that Lhx2 expression is necessary to ensure the correct expression of Robol and Robo2 receptors in developing thalamic neurons.

\section{Deletion of $L h x 2$ causes axon guidance defects in thalamocortical axons}

We have shown that the Lhx2 protein is dynamically regulated in postmitotic thalamic neurons and, while it is downregulated in rTh and iTh thalamic-projecting neurons during axonal projection, Lhx2 is expressed strongly in neurons forming cTh nuclei. However, a high percentage of thalamic neurons express strong levels of this transcription factor at early postmitotic stages. Thus, high levels of Lhx2 before axon outgrowth may be necessary for specific axon guidance programs to unfold. We hypothesized that the loss of Lhx2 function would compromise the guidance of these axons, particularly in the MGv where Lhx2 protein remains abundant during axon pathfinding. At E14.5, GFP-positive TCAs began to arrive at the neocortex, running through the intermediate zone and subplate areas but remaining outside the cortical plate $(n=4$; Fig. $7 B-C$ '), which is invaded by axons around E18.5 (López-Bendito and Molnár, 2003). Interestingly, TCAs that lack Lhx2 prematurely invaded the cortical plate and this error can be observed along several rostrocaudal cortical levels, suggesting that distinct populations of thalamic neurons are similarly affected by the lack of $\operatorname{Lhx} 2\left(n=4\right.$; Fig. $7 D-E^{\prime}$; data not shown). These results highlight the need for this transcription factor to regulate several aspects of the development of these connections. This premature invasion in Th-Lhx2 mice suggests that the rostrocaudal dispersion of TCAs in the neocortex is perturbed in the absence of Lhx2, resulting in the aberrant targeting of TCAs to cortical areas. However, because Lhx2 was removed in the ThLhx2 mice at early stages, we tested whether these changes could be due to alterations in the process by which thalamic structures become differentiated. The gross expression pattern for both $N g n 2$ and Gbx2 transcription factors were not changed in ThLhx2 mutant embryos $(n=3$; Fig. $7 F-O)$. Moreover, the principal thalamic nuclei, as the MGv, dLG, and VP, were present in Th-Lhx2, as delineated by the Ngn2 and Gbx2 expression (Fig. 7F-O; Nakagawa and O'Leary, 2001; Chen et al., 2009).

Next, we studied TCA topography at later developmental stages when TCAs have already arrived to their correct cortical targets (Fig. 8 A) (López-Bendito and Molnár, 2003). Because the MGv nucleus of the cTh maintain high levels of Lhx2 during all embryonic stages studied, we focused on TCAs projecting to occipital cortical areas. DiI crystals were placed in the ventral occipital cortex (putative primary auditory area) and DiA in the parietal cortex (putative primary somatosensory area) of E17.5 wild-type and Th-Lhx2 mutant embryos. TCAs that reach the visual cortical area pass through the underlying white matter of the auditory cortical region. As such, injections in the auditory cortex generally result in the back-labeling of both visual and auditory thalamic nuclei. Indeed, in both wild-type and Th-Lhx2 embryos, retrograde DiI labeling of the dLG and DiA in the VP was evident at intermediate thalamic levels ( $n=5$ wild type; $n=$ 6 Th-Lhx2; Fig. $\left.8 B, B^{\prime}, D, D^{\prime}\right)$. However, at caudal levels, some neurons in the most caudal VP nucleus were also back-labeled by ventral occipital DiI injections in Th-Lhx2 mutant mice, indicating that some intermediate TCAs abnormally target the putative auditory cortex (Fig. 8E, E'). Interestingly, no back-labeled cells were found in the putative MGv nucleus of the thalamus in ThLhx2 embryos ( $n=5$ wild type; $n=6$ Th-Lhx2; Fig. $8 C, C^{\prime}, E, E^{\prime}$ ), demonstrating that these neurons do not project to their normal cortical target area in the absence of Lhx2. DiI injections in the putative $\mathrm{MGv}$ nucleus revealed a strong reduction in the number of TCA axons that reached caudal cortical areas in the absence of Lhx2 (data not shown). It should be noted that MGv nucleus was smaller in Th-Lhx2 mice than in their wild-type counterparts (Fig. 7O), suggesting that Lhx2 may also be involved in the development of this nucleus.

Robo functions downstream of $L h x 2$ to control TCA guidance Both overexpression of Lhx2 in the embryonic thalamus (Fig. 3) and the absence of Robo receptors (López-Bendito et al., 2007) significantly alter TCA pathfinding, suggesting that Lhx2 may control TCA guidance by regulating Robo levels in vivo. We hypothesized that the increase in Robol and/or Robo2 signaling in rTh- and iTh-projecting neurons expressing low levels of Lhx2 is a key step in producing a correct axonal pathfinding through the diencephalon. To determine to what extent Lhx2 activity influences TCA guidance via Robo receptors, we quantified the invasion of hypothalamic structures in thalamic neurons coelectroporated with $L h x 2$ and a full-length Robol receptor (Robo1-FL) at E12.5. Remarkably, in contrast to the abnormal axonal invasion of hypothalamic areas seen following $L$ hx2 electroporation alone, TCA extension following co-electroporation with $L h \times 2$ and Robo1-FL did not differ significantly from that in controls electroporated with Gfp ( $L h \times 2: 0.61 \pm 0.05, n=12$; Robo1-FL+Lhx2: $0.41 \pm 0.03, n=10 ; G f p: 0.37 \pm 0.03, n=16$; Fig. $9 A-D)$. Accordingly, the TCAs from neurons electroporated with plasmids expressing $L h \times 2$ and Robo1-FL extended significantly farther in the cortex than those electroporated with plasmids expressing $L h x 2$ alone. These results indicate that augmenting Robol function can rescue the axon guidance defect at the hypothalamic area provoked by the overexpression of Lhx2 activity in vivo. To summarize, our results demonstrate that Lhx2 influences key aspects of TCA development in a cell-autonomous manner, affecting topographic specification and cortical targeting.

\section{Discussion}

In the present study, we describe the dynamic regulation of the LIM-HD transcription factor Lhx 2 in postmitotic thalamic neurons. While a large percentage of thalamic neurons express Lhx2 at early postmitotic stages, only in specific nuclei of caudal regions do thalamocortical neurons maintain high levels of Lhx2 protein during axonal pathfinding (Fig. 9E). In contrast, Lhx2 expression is quickly downregulated in rostral and intermediate thalamic-projecting neurons as they extend axons toward the cortex. Conditional deletion of Lhx 2 in the developing thalamus provokes premature entry of these axons into the cortical plate of the neocortex and abnormal targeting by TCAs (Fig. 9E). We also 
A
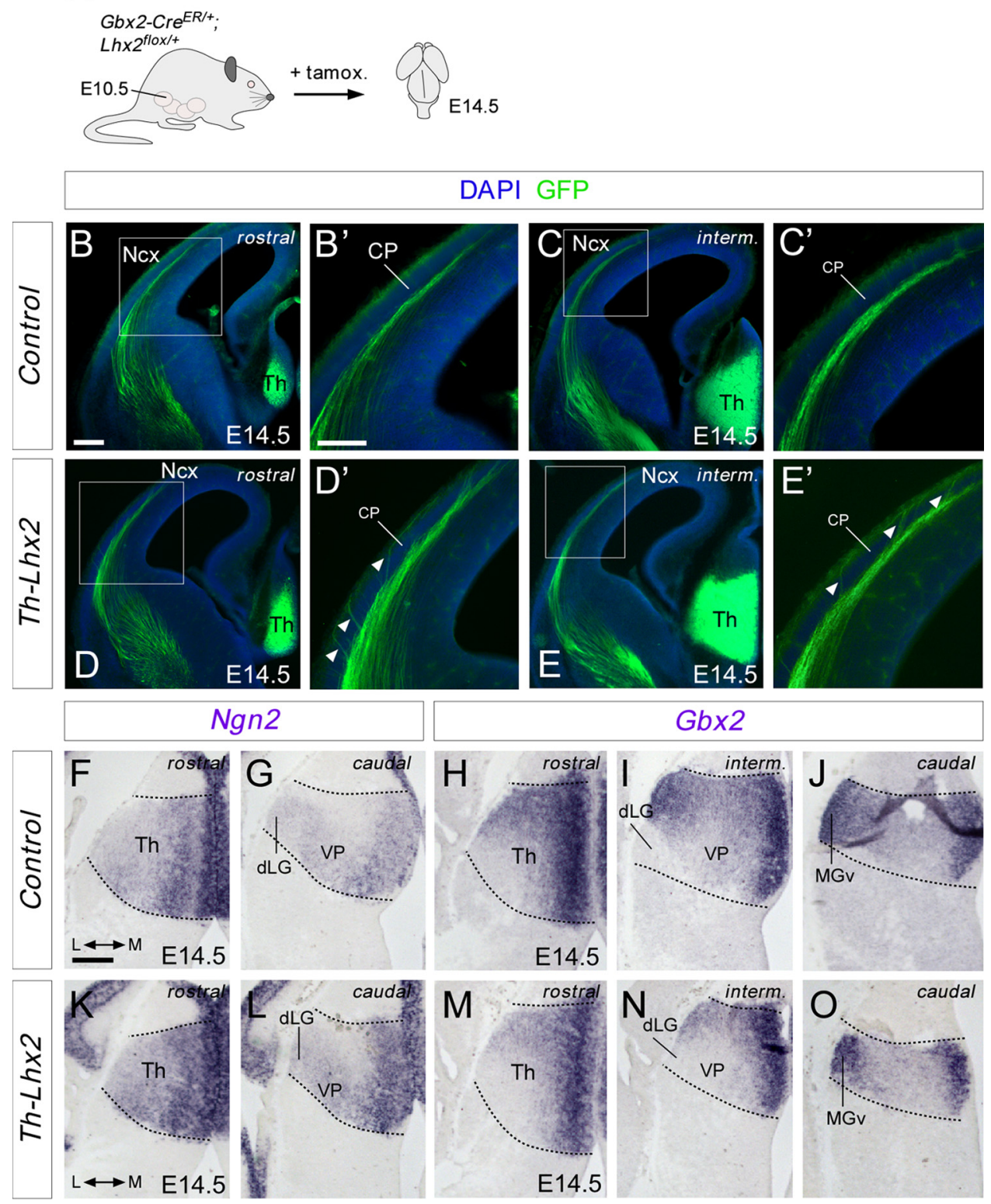

Figure 7. Conditional deletion of $L h \times 2$ in thalamic neurons provokes premature invasion of the cortical plate. $A$, Schematic diagram of the strategy used to delete $L h \times 2$ in the thalamus at specific developmental stages. $\boldsymbol{B}-\boldsymbol{E}^{\prime}$, Rostromedial coronal sections showing GFP immunohistochemistry in control (B-C $\left.\boldsymbol{C}^{\prime}\right)$ and Th-Lhx2 (D-E) brains at E14.5. TCAs invaded the cortical plate (CP) abnormally at these early embryonic stages ( $\boldsymbol{D}^{\prime}, \boldsymbol{E}^{\prime}$, arrowheads). $\boldsymbol{F}, \mathbf{0}, \mathbf{I n}$ situ hibridization for $N g n 2$ and $G b x 2$ at E14.5 embryonic brains, showing that there is no difference in the thalamic patterning between the control $(\boldsymbol{F}-\boldsymbol{J})$ and the Th-Lhx2 $(\boldsymbol{K}-\mathbf{0})$ brains. Th, thalamus; Ncx, neocortex. Scale bars: (in $\boldsymbol{B}) \boldsymbol{B}, \boldsymbol{C}, \boldsymbol{D}, \boldsymbol{E}, 300 \mu \mathrm{m}$; (in $\left.\boldsymbol{B}^{\prime}\right) \boldsymbol{B}^{\prime}, \boldsymbol{C}^{\prime}, \boldsymbol{D}^{\prime}, \boldsymbol{E}^{\prime}, 200 \mu \mathrm{m}$; (in $\left.\boldsymbol{F}\right) \boldsymbol{F}-\mathbf{0}, 200 \mu \mathrm{m}$.

describe a downstream mechanism involving the potentially direct repression of the Robo1 and Robo 2 guidance receptors by this transcription factor.

Dynamic regulation of $\mathrm{Lhx} 2$ in postmitotic thalamic neurons The expression of distinct transcription factors, including Lhx2 and Lhx9 of the LIM-HD family, has been shown to segregate the nuclei in the developing thalamus, suggesting that differential cellautonomous activities direct TCA development (Nakagawa and O'Leary, 2001). We describe the dynamic regulation of Lhx2 in postmitotic rostrocaudal thalamic neurons, as evident by Lhx2 immunohistochemistry. Using BrdU pulse experiments, we demonstrate that a large percentage of thalamic neurons accumulate high levels of Lhx2 16-18 h after their birth. As these postmitotic cells (Lhx2- positive) migrate laterally to their final position, they rapidly downregulate Lhx2 in rostral and intermediate thalamic structures at the time they undergo axonal pathfinding, whilst it is maintained in neurons that form the caudal nuclei, such as the MGv nucleus. This pattern is consistent with previous findings describing weak Lhx2 mRNA expression in rostromedial thalamic nuclei, including the dLG and VP nuclei (Nakagawa and O'Leary, 2001). Interestingly, a graded distribution of Lhx2 mRNA and protein has been also demonstrated in the developing cortical ventricular zone, which is essential for the formation of cortical structures (Porter et al., 1997; Donoghue and Rakic, 1999; Nakagawa et al., 1999; Rétaux et al., 1999; Bulchand et al., 2003). Moreover, Bmp4 and Bmp2-derived molecules from the roof plate are shown to regulate Lhx2 cortical gradient (Monuki et al., 2001). Bmp morfogenes, particularly Bmp7, 


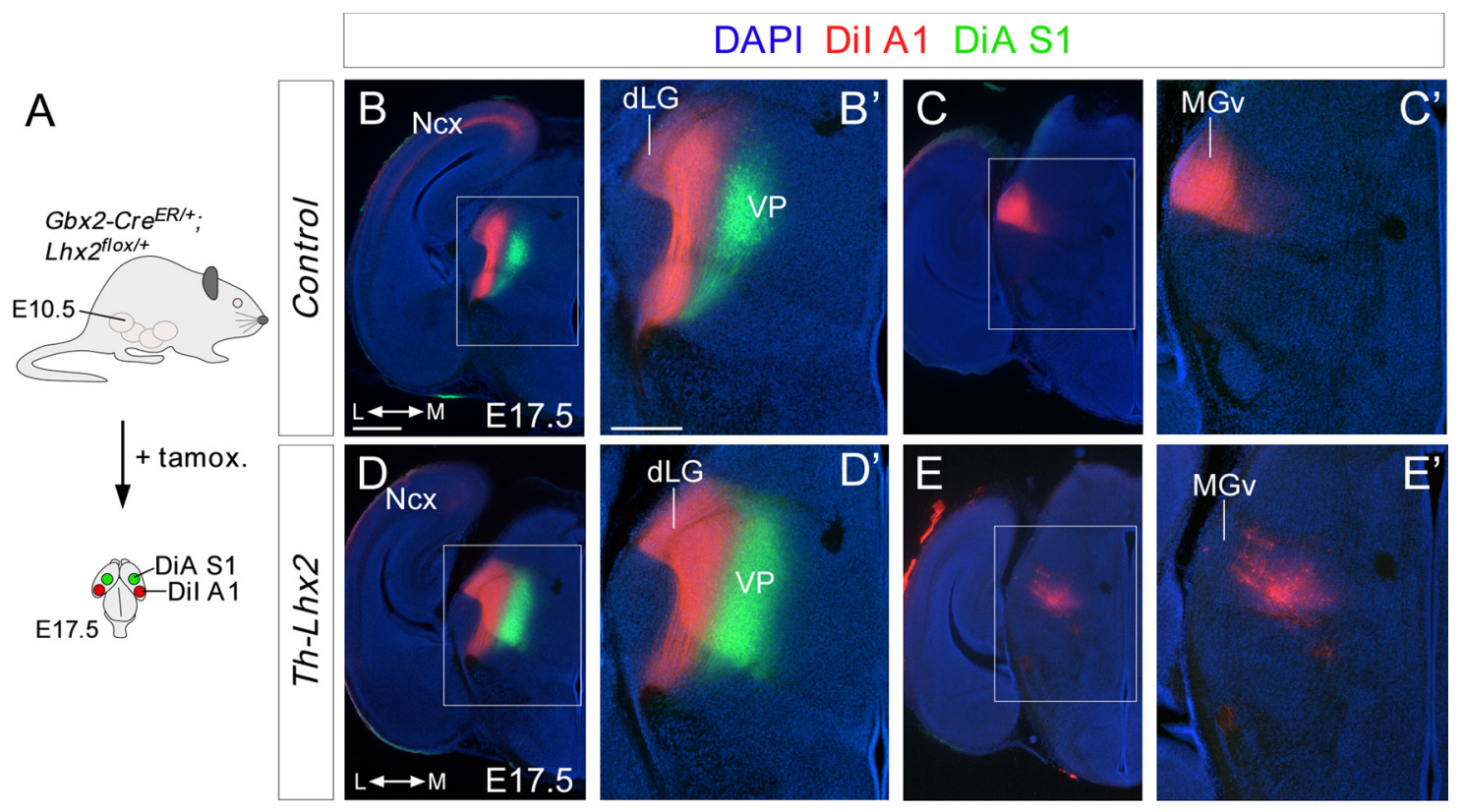

Figure 8. Tracing experiments revealed topographical thalamocortical defects after the conditional deletion of $L h x 2$ in the thalamus. $A$, Schematic diagram illustrating the strategy used to conditionally delete Lhx2 by tamoxifen administration and the dye-tracing studies performed. $\boldsymbol{B}-\boldsymbol{E}^{\prime}$, Coronal sections showing retrograde-labeled cells in the distinct thalamic nuclei after Dil injection (red) into the A1 cortical area and DiA (green) into the S1 cortical area of control ( $\boldsymbol{B}-\boldsymbol{C})$ and $T h$-Lhx2 ( $\boldsymbol{D}-\boldsymbol{E}$ ) embryos. After injection in the A1, retrograde-labeled cells were observed in the $\mathrm{dLG}$ and MGv nuclei of control brains. However, no retrograde-labeled cells were observed in the MGv of Th-Lhx2 mice and abnormal retrograde labeling was observed in some ectopic thalamic neurons $\left(\boldsymbol{E}-\boldsymbol{E}^{\prime}\right)$ in the most caudal part of the VP nucleus. Scale bars: (in $\left.\boldsymbol{B}\right) \boldsymbol{B}, \boldsymbol{C}, \boldsymbol{D}, \boldsymbol{E}, 300 \mu \mathrm{m}$; (in $\left.\boldsymbol{B}^{\prime}\right) \boldsymbol{B}^{\prime}, \boldsymbol{C}^{\prime}, \boldsymbol{D}^{\prime}, \boldsymbol{E}^{\prime}, 200 \mu \mathrm{m}$.

are also expressed in developing thalamic neurons (Suzuki-Hirano et al., 2011), suggesting that a similar mechanism might establish the Lhx2 expression pattern observed.

Combining immunohistochemistry against Lhx2 with backlabeling from principal cortical areas, we showed that MGv thalamic neurons maintain high levels of Lhx2 while projecting to A1. However, dLG, VB, and VL neurons have very low levels of expression of Lhx2 when connecting to their reciprocal targets, V1, S1, and M1, respectively. Interestingly, $L h x 2$ expression also shows a correlation between these nuclei and the corresponding cortical areas. Whereas visual, motor, and somatosensory areas do not express $L h \times 2$ or express very low levels, the auditory area, target of MGv axons, expresses the highest level of $L h \times 2$ (Nakagawa et al., 1999). Thus, it is possible that Lhx2 may influence the specific targeting of TCAs to distinct cortical areas through the precise regulation of downstream guidance receptors.

\section{Lhx2 influences TCA pathfinding and topography in a cell-autonomous manner}

It is well recognized that the initial topography of TCAs is acquired in the ventral telencephalon (vTel) in response to gradients of axon guidance cues in this region (Métin and Godement, 1996; Dufour et al., 2003; Garel and Rubenstein, 2004; Vanderhaeghen and Polleux, 2004; Bielle et al., 2011). Thus, we expect that cell-autonomous and non-autonomous mechanisms, such as the specific expression of guidance receptors by TCAs or key interaction with intermediate targets, play a role in sorting TCAs before they enter the neocortex. By conditionally deleting Lhx2 in thalamic neurons in vivo, we identified the transcription factor $\mathrm{Lhx} 2$ as a cell-autonomous regulator of key aspects of TCA connectivity.

Lhx2-null mutant mice exhibit severe defects in the development of the thalamocortical pathway without affecting the overall patterning of thalamic structures (Lakhina et al., 2007). Dye-tracing studies revealed that TCAs fail to arrive to the cortex at E14.5 and they descend abruptly into the vTel. As $L h \times 2$ mutant mice die early in embryogenesis, analyzing the role of Lhx2 in the guidance and topography of thalamocortical connectivity at later stages is impossible in vivo. Nevertheless, using ex vivo cocultures from wild-type and Lh $\times 2$ mutant mice, it was concluded that $L h \times 2$ activity is required in the vTel but not for the correct guidance of TCAs in the thalamus (Lakhina et al., 2007). Together with the defects observed following the conditional deletion of Lhx2, our gain-of-function experiments in thalamic neurons demonstrate that this transcription factor also plays a cell-autonomous role in regulating distinct aspects of thalamocortical development. The differences in the defects observed upon conditional deletion of $L h \times 2$ and those seen in the null mutant suggests a dual function of Lhx 2 in thalamocortical connectivity, possibly involving the regulation of several processes that depend on cofactor association. Moreover, the ex vivo coculture experiments performed by Lakhina and colleagues (2007) used thalamus at a stage (E14.5) when thalamic axons have already reached the cortex. Our manipulation of Lhx2 in thalamic neurons was done at E12.5, when TCAs are being specified and when the manipulation of Lhx2 may have a bigger impact in TCA pathfinding. In any case, our results demonstrate that Lhx2 differentially regulates the guidance of axons from distinct populations of thalamic neurons, depending on the levels of Lhx2 they express and, in turn, on the levels of Robo1 and Robo2. Meanwhile, Lhx2 expression in progenitor cells of the vTel affects the development of TCAs by specifying the formation of intermediate targets, such as internal capsule cells, which are crucial for continued progression toward the cortex (Mitrofanis and Guillery, 1993; Molnár and Cordery, 1999; Lakhina et al., 2007). In fact, our study demonstrates that Lhx2 is a repressor of Robo1 and Robo2 receptors. However, it remains unclear whether the higher levels of Robo1 and Robo2 receptors in TCAs of Th-Lhx2 mutant mice underlie the defects in axon guidance and topography observed. Interestingly, we recently showed that Slit/ Robo interactions are necessary for the correct initial topographical sorting of TCAs in the ventral telencephalon (Bielle et al., 2011). Therefore, modulating Robo receptor levels through Lhx2 function 


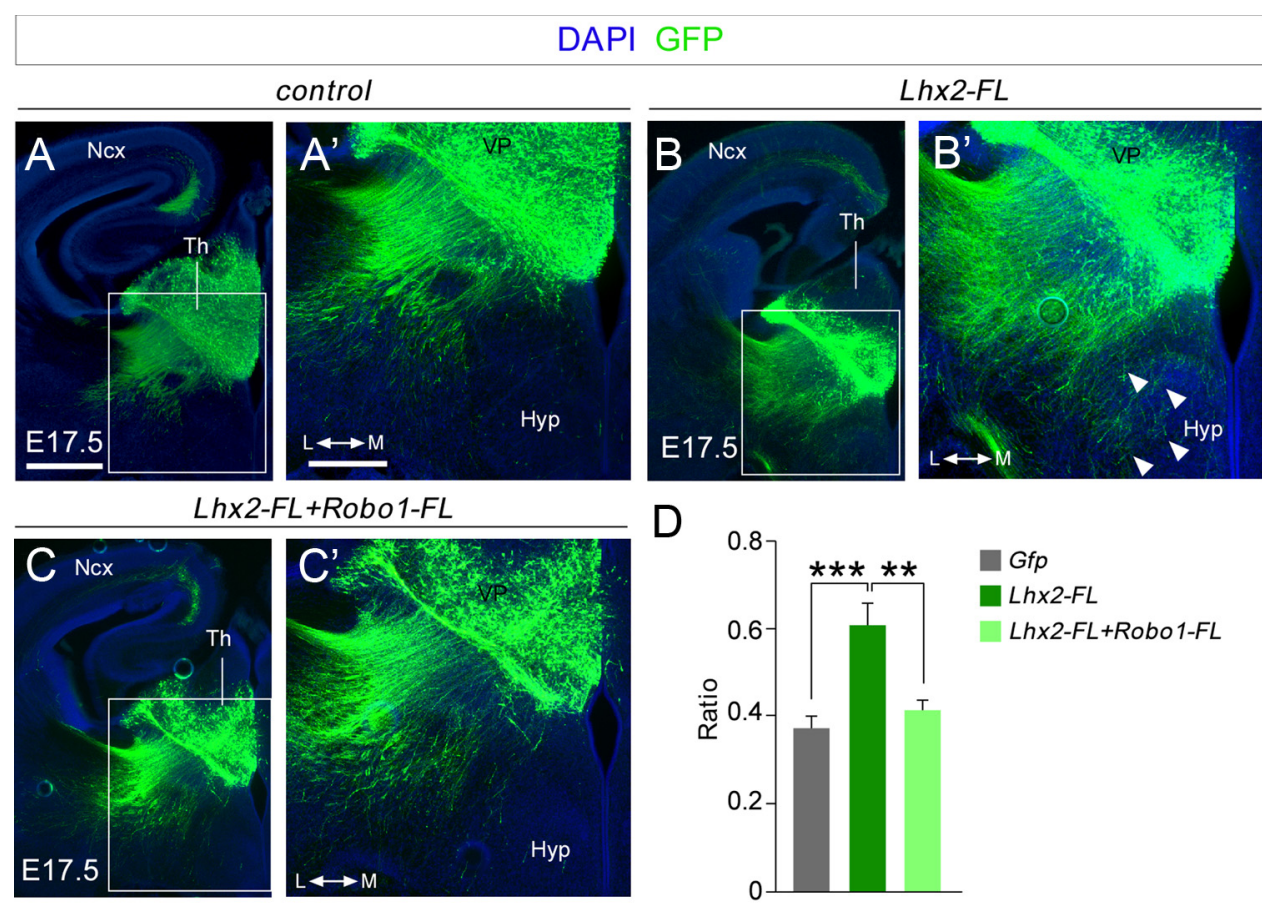

E
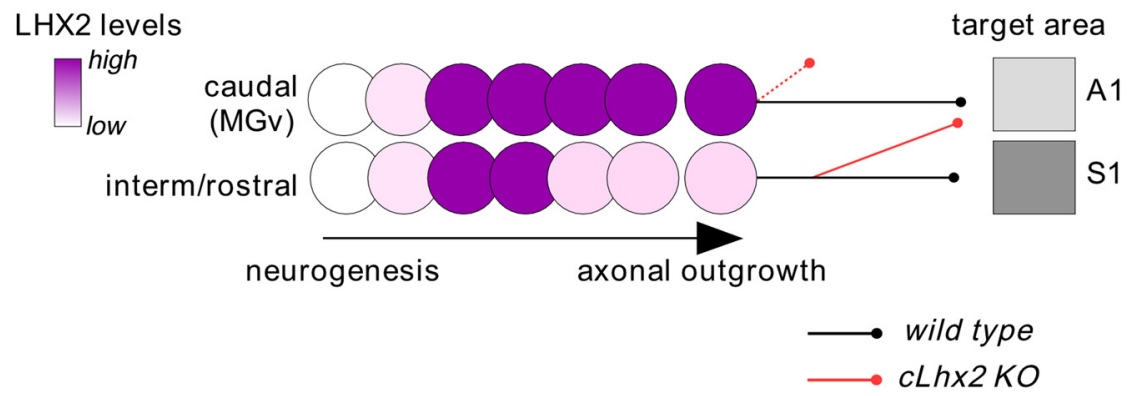

Figure 9. Coelectroporation of $L h \times 2$ and Robo1 in the thalamus rescues the axon guidance phenotype of thalamocortical axons. $\boldsymbol{A}-\boldsymbol{C}^{\prime}$, Electroporated brains with $G f p(\boldsymbol{A}, \boldsymbol{A})$, $L h \times 2(\boldsymbol{B}, \boldsymbol{B})$, and Lhx2 + Robo1 (C, C), respectively, showing the GFP-positive axons that were electroporated. Axons overexpressing Lhx2 and Robo1 follow a normal pathway toward the cortex as control experiments. $\boldsymbol{D}$, Quantification of the data shown in $\boldsymbol{A}^{\prime}, \boldsymbol{B}^{\prime}$, and $\boldsymbol{C}^{\prime}$ at the hypothalamic region as performed in Figure 3. Asterisks indicate significance at ${ }^{* *} p<0.01$ and ${ }^{* * *} p<0.001$, one-way ANOVA test with Tukey's posthoc analysis. $E$, Schematic diagram showing the dynamic regulation of Lhx2 protein in postmitotic thalamic neurons at rostrocaudal levels in relation to $T C A$ pathfinding. Ncx, neocortex; Hyp, hypothalamus. Scale bars: (in $\boldsymbol{A}) \boldsymbol{A}, \boldsymbol{B}, \boldsymbol{C} ; 300 \mu \mathrm{m}$; (in $\left.\boldsymbol{A}^{\prime}\right) \boldsymbol{A}^{\prime}, \boldsymbol{B}^{\prime}, \boldsymbol{C}^{\prime}, 200 \mu \mathrm{m}$.

in distinct TCAs would appear to be a plausible mechanism by which the precise positioning of TCAs is achieved.

\section{Robo1 and Robo2 receptors are potentially direct downstream targets of $\mathrm{Lhx} 2$ function}

Using a transgenic mouse line in which Lhx2 is deleted only in the thalamus, we found a significant increase in the thalamic expression of Robo1 and Robo2, revealed by both quantitative PCR and in situ hybridization. This increase in both Robo receptors is also found in the Lhx2-null mutant mice. Furthermore, overexpression of Lhx2 in rostral and intermediate thalamic neurons by in utero electroporation results in the abnormal invasion of the hypothalamus by electroporated axons, consistent with the finding that normal Slit/Robo signaling is crucial to correctly channel TCAs toward the cortex (Andrews et al., 2006; López-Bendito et al., 2007). While the expression patterns of Lhx2 and Robo2 suggest that the former represses the latter, Lhx2 appears to positively regulate Robol given the expression of both factors in similar regions of the thalamus. This apparent finding might be explained by Lhx2 exerting weaker repression on Robo1 than on Robo2, as suggested by our quantitative PCR results in both overexpressing experiments in wild-type thalamus and the analysis of Robo1 and Robo2 activation in Th-Lhx2 mutant. Alternatively, Robol expression may be positively regulated by Lhx9, with which it also shares a similar pattern of expression (data not shown), and Lhx9 may in turn be downregulated by Lhx2 gain of function. Finally, although $L h \times 2$ and Robol expression overlaps in the thalamic regions, it remains to be determined whether both genes colocalize at a single-cell level. In any case, gain of function of Robol fully rescues the axon guidance defects produced by overexpression of Lhx2, demonstrating that Robo1 receptors are potentially direct downstream mediators of Lhx2 function in TCA guidance.

In ChIP assays, Lhx2 appears to bind to conserved regulatory sequences in the Robol and Robo 2 genes in thalamic neurons. However, EMSA assays suggest that, in the spinal cord, Lhx2 binds directly to the Rig-1 gene but not to Robo1 and Robo2 (Wilson et al., 2008). We did not detect Rig-1 mRNA or protein in the thalamus during TCA pathfinding. In fact, in our ChIP assays, Lhx2 did not appear to bind to the Rig-1 gene in thalamic neurons, in contrast to spinal cord tissue. These results suggest that, while commissural neurons depend on Rig-1 for their axons to cross the midline (Sabatier et al., 2004), thalamocortical neurons do not require Rig-1 for their axons to extend into the cortex. Future experiments (e.g., by lu- 
ciferase assay) will be needed to get a definite answer on whether Lhx2 directly regulates Robo receptors in thalamic neurons. To summarize, the Lhx2 gene is a key regulator of axon guidance and topographic targeting relevant for brain wiring. These developmental events specify the functions of distinct pools of thalamic neurons, particularly those related to caudal thalamic structures.

\section{References}

Andrews W, Liapi A, Plachez C, Camurri L, Zhang J, Mori S, Murakami F, Parnavelas JG, Sundaresan V, Richards LJ (2006) Robol regulates the development of major axon tracts and interneuron migration in the forebrain. Development 133:2243-2252.

Angevine JB Jr (1970) Time of neuron origin in the diencephalon of the mouse. An autoradiographic study. J Comp Neurol 139:129-187.

Bach I (2000) The LIM domain: regulation by association. Mech Dev 91:5-17. Bagri A, Marín O, Plump AS, Mak J, Pleasure SJ, Rubenstein JL, TessierLavigne M (2002) Slit proteins prevent midline crossing and determine the dorsoventral position of major axonal pathways in the mammalian forebrain. Neuron 33:233-248.

Bielle F, Marcos-Mondéjar P, Leyva-Díaz E, Lokmane L, Mire E, Mailhes C, Keita M, García N, Tessier-Lavigne M, Garel S, López-Bendito G (2011) Emergent growth cone responses to combinations of slit 1 and netrin 1 in thalamocortical axon topography. Curr Biol 21:1748-1755.

Bulchand S, Subramanian L, Tole S (2003) Dynamic spatiotemporal expression of LIM genes and cofactors in the embryonic and postnatal cerebral cortex. Dev Dyn 226:460-469.

Bulfone A, Puelles L, Porteus MH, Frohman MA, Martin GR, Rubenstein JL (1993) Spatially restricted expression of $D l x-1, D l x-2$ (Tes-1), Gbx-2, and Wnt-3 in the embryonic day 12.5 mouse forebrain defines potential transverse and longitudinal segmental boundaries. J Neurosci 13:3155-3172.

Chen L, Guo Q, Li JY (2009) Transcription factor Gbx2 acts cellnonautonomously to regulate the formation of lineage-restriction boundaries of the thalamus. Development 136:1317-1326.

Chou SJ, Perez-Garcia CG, Kroll TT, O'Leary DD (2009) Lhx2 specifies regional fate in Emx1 lineage of telencephalic progenitors generating cerebral cortex. Nat Neurosci 12:1381-1389.

Donoghue MJ, Rakic P (1999) Molecular gradients and compartments in the embryonic primate cerebral cortex. Cereb Cortex 9:586-600.

Dufour A, Seibt J, Passante L, Depaepe V, Ciossek T, Frisén J, Kullander K, Flanagan JG, Polleux F, Vanderhaeghen P (2003) Area specificity and topography of thalamocortical projections are controlled by ephrin/Eph genes. Neuron 39:453-465.

Garel S, Rubenstein JL (2004) Intermediate targets in formation of topographic projections: inputs from the thalamocortical system. Trends Neurosci 27:533-539.

Garel S, Yun K, Grosschedl R, Rubenstein JL (2002) The early topography of thalamocortical projections is shifted in Ebf1 and Dlx1/2 mutant mice. Development 129:5621-5634.

Jones EG, Rubenstein JL (2004) Expression of regulatory genes during differentiation of thalamic nuclei in mouse and monkey. J Comp Neurol 477:55-80.

Jones L, López-Bendito G, Gruss P, Stoykova A, Molnár Z (2002) Pax6 is required for the normal development of the forebrain axonal connections. Development 129:5041-5052.

Jurata LW, Thomas JB, Pfaff SL (2000) Transcriptional mechanisms in the development of motor control. Curr Opin Neurobiol 10:72-79.

Kania A, Jessell TM (2003) Topographic motor projections in the limb imposed by LIM homeodomain protein regulation of ephrin-A:EphA interactions. Neuron 38:581-596.

Kania A, Johnson RL, Jessell TM (2000) Coordinate roles for LIM homeobox genes in directing the dorsoventral trajectory of motor axons in the vertebrate limb. Cell 102:161-173.

Lakhina V, Falnikar A, Bhatnagar L, Tole S (2007) Early thalamocortical tract guidance and topographic sorting of thalamic projections requires LIM-homeodomain gene Lhx2. Dev Biol 306:703-713.

Lee SK, Pfaff SL (2001) Transcriptional networks regulating neuronal identity in the developing spinal cord. Nat Neurosci 4 [Suppl]:1183-1191.

López-Bendito G, Molnár Z (2003) Thalamocortical development: how are we going to get there? Nat Rev Neurosci 4:276-289.
López-Bendito G, Chan CH, Mallamaci A, Parnavelas J, Molnár Z (2002) Role of Emx2 in the development of the reciprocal connectivity between cortex and thalamus. J Comp Neurol 451:153-169.

López-Bendito G, Cautinat A, Sánchez JA, Bielle F, Flames N, Garratt AN, Talmage DA, Role LW, Charnay P, Marín O, Garel S (2006) Tangential neuronal migration controls axon guidance: a role for neuregulin-1 in thalamocortical axon navigation. Cell 125:127-142.

López-Bendito G, Flames N, Ma L, Fouquet C, Di Meglio T, Chedotal A, Tessier-Lavigne M, Marín O (2007) Robol and Robo2 cooperate to control the guidance of major axonal tracts in the mammalian forebrain. J Neurosci 27:3395-3407.

Mangale VS, Hirokawa KE, Satyaki PR, Gokulchandran N, Chikbire S, Subramanian L, Shetty AS, Martynoga B, Paul J, Mai MV, Li Y, Flanagan LA, Tole S, Monuki ES (2008) Lhx2 selector activity specifies cortical identity and suppresses hippocampal organizer fate. Science 319:304-309.

Métin C, Godement P (1996) The ganglionic eminence may be an intermediate target for corticofugal and thalamocortical axons. J Neurosci 16:3219-3235.

Mitrofanis J, Guillery RW (1993) New views of the thalamic reticular nucleus in the adult and the developing brain. Trends Neurosci 16:240-245.

Molnár Z, Cordery P (1999) Connections between cells of the internal capsule, thalamus, and cerebral cortex in embryonic rat. J Comp Neurol 413:1-25.

Molnár Z, Higashi S, López-Bendito G (2003) Choreography of early thalamocortical development. Cereb Cortex 13:661-669.

Monuki ES, Porter FD, Walsh CA (2001) Patterning of the dorsal telencephalon and cerebral cortex by a roof plate-Lhx2 pathway. Neuron 32:591-604.

Nakagawa Y, O'Leary DD (2001) Combinatorial expression patterns of LIM-homeodomain and other regulatory genes parcellate developing thalamus. J Neurosci 21:2711-2725.

Nakagawa Y, Johnson JE, O'Leary DD (1999) Graded and areal expression patterns of regulatory genes and cadherins in embryonic neocortex independent of thalamocortical input. J Neurosci 19:10877-10885.

Porter FD, Drago J, Xu Y, Cheema SS, Wassif C, Huang SP, Lee E, Grinberg A, Massalas JS, Bodine D, Alt F, Westphal H (1997) Lhx2, a LIM homeobox gene, is required for eye, forebrain, and definitive erythrocyte development. Development 124:2935-2944.

Pratt T, Quinn JC, Simpson TI, West JD, Mason JO, Price DJ (2002) Disruption of early events in thalamocortical tract formation in mice lacking the transcription factors Pax6 or Foxg1. J Neurosci 22:8523-8531.

Rétaux S, Rogard M, Bach I, Failli V, Besson MJ (1999) Lhx9: a novel LIMhomeodomain gene expressed in the developing forebrain. J Neurosci 19:783-793.

Sabatier C, Plump AS, Le M, Brose K, Tamada A, Murakami F, Lee EY, Tessier-Lavigne M (2004) The divergent Robo family protein rig-1/ Robo3 is a negative regulator of slit responsiveness required for midline crossing by commissural axons. Cell 117:157-169.

Seibt J, Schuurmans C, Gradwhol G, Dehay C, Vanderhaeghen P, Guillemot F, Polleux F (2003) Neurogenin2 specifies the connectivity of thalamic neurons by controlling axon responsiveness to intermediate target cues. Neuron 39:439-452.

Sharma K, Sheng HZ, Lettieri K, Li H, Karavanov A, Potter S, Westphal H, Pfaff SL (1998) LIM homeodomain factors Lhx3 and Lhx4 assign subtype identities for motor neurons. Cell 95:817-828.

Suzuki-Hirano A, Ogawa M, Kataoka A, Yoshida AC, Itoh D, Ueno M, Blackshaw S, Shimogori T (2011) Dynamic spatiotemporal gene expression in embryonic mouse thalamus. J Comp Neurol 519:528-543.

Törnqvist G, Sandberg A, Hägglund AC, Carlsson L (2010) Cyclic expression of lhx2 regulates hair formation. PLoS Genet 6:e1000904.

Tuttle R, Nakagawa Y, Johnson JE, O’Leary DD (1999) Defects in thalamocortical axon pathfinding correlate with altered cell domains in Mash-1deficient mice. Development 126:1903-1916.

Vanderhaeghen P, Polleux F (2004) Developmental mechanisms patterning thalamocortical projections: intrinsic, extrinsic and in between. Trends Neurosci 27:384-391.

Wilson SI, Shafer B, Lee KJ, Dodd J (2008) A molecular program for contralateral trajectory: Rig-1 control by LIM homeodomain transcription factors. Neuron 59:413-424. 